\title{
El fenómeno de mortalidad por suicidio en México: patrones territoriales a partir de 20 años de información
}

\section{Suicide mortality phenomenon in México: territorial patterns based on 20 years of information}

José Luis Manzanares Rivera

Profesor investigador. Departamento de Estudios Urbanos y Medio Ambiente. El Colegio de la Frontera Norte. Av. Reforma, Local 1, 2 y 3 (Centro Corporativo Norte) Col. del Rosario, (84020), Nogales, Son, México, jlmanzanares@colef.mx, ORCID https://orcid.org/0000-0003-3394-4967

Recibido: 20 de noviembre 2019 || Aprobado: 10 de julio 2020

Resumen

El objetivo es documentar la evolución temporal y distribución territorial del suicidio en México durante el periodo 1998-2017 con énfasis en el caso del estado de Aguascalientes. La metodología considera análisis exploratorio de datos utilizando registros públicos de mortalidad provenientes del sistema nacional de información en salud a partir de dos estructuras de datos: corte transversal y series de tiempo. Los resultados indican que los estados de Chihuahua y Aguascalientes son los de mayor mortalidad actualmente. Se documentó un patrón temporal de mayor incidencia mensual para mayo y junio en el periodo de 20 años estudiado. Por su parte el análisis a escala de zona Metropolitana permite identificar a la ZM de Aguascalientes como la de mayor concentración específicamente afectando a la población entre los 12 y 32 años principalmente.

Palabras clave: Suicidio; Hacinamiento; Crecimiento económico; México

\section{Abstract}

The purpose is to document Mexico's temporary suicide evolution during the period 1998-2017 with a focus on Aguascalientes State. The methodology is based on exploratory data analysis using public mortality records collected by the national system for health information for two different data structures, cross section and time series. Results indicate that the States of Chihuahua and Aguascalientes are the ones with the highest mortality currently. A temporal pattern of higher monthly incidence was documented for May and June in the 20-year period studied. For its part, the analysis at the metropolitan area scale, allows to identify the Aguascalientes Metropolitan area as the one with the highest concentration, specifically affecting the population between 12 and 32 years of age.

Key words: Suicide; Overcrowding; Economic growth; México

Cita sugerida: Manzanares Rivera, J.L. (2020). El fenómeno de mortalidad por suicidio en México: patrones territoriales a partir de 20 años de información. Estudios Socioterritoriales. Revista de Geografía, (28), 059. DOI: https://doi.org/10.37838/unicen/est.28-059 (c) (i) (2) (2) 
Muy a menudo los profesionales de la salud se concentran en lo que es más simple, es decir, tratar una condición mental o médica, mientras enfrentar los riesgos del suicido rebasa el enfoque tradicional e involucra el entendimiento humano del sufrimiento (Pompili, 2018) (traducción nuestra)

\section{INTRODUCCIÓN}

Acorde con las tasas de mortalidad ajustadas por estructura de edad y sexo, durante los últimos 20 años, el suicidio como causa de muerte en México registró un crecimiento del $185 \%$ para el sexo femenino y del $50 \%$ para el sexo masculino, al pasar de seis individuos por cada cien mil habitantes en 1998, a nueve en el año 2017; comportamiento que superó la dinámica observada por la tasa de mortalidad en el país, cuyo crecimiento alcanzó un $30 \%$ durante el mismo periodo.

Los avances sobre el estudio de este fenómeno que se manifiesta cada vez con mayor frecuencia a escala global (OPS, 2016), indican qué factores sociales son fundamentales para comprender la ocurrencia del suicidio (Abrutyn y Mueller, 2014), un fenómeno de salud pública cuyas causas se extienden más allá de los síntomas identificados desde la aproximación clínica.

Así, por ejemplo, el grado de conectividad social es un factor que ha sido identificado al estudiar las causas del suicidio. De acuerdo con Joiner (2007) y Van Orden et al. (2010) los desequilibrios asociados a este dominio pueden considerarse como expresiones de necesidades humanas no satisfechas vinculadas con el sentido de pertenencia y constituyen motivos comunes que subyacen a las diversas expresiones de este fenómeno.

La importancia de contar con estudios que analicen este fenómeno, se debe en parte a que se trata de una causa de muerte con impactos sociales severos por la pérdida prematura de vidas. Por su parte, la Organización Mundial de la Salud documenta que las causas incluyen afecciones clínicas extensamente documentadas sobre la salud mental como la condición de depresión (WHO, 2018).

Como se ha documentado por estudios que abordan la distribución espacial de las condiciones de salud pública, México es un país con un elevado grado de diversidad (Rosales, Carvajal y de Zapien, 2016), rasgo que impacta también en los procesos de salud-enfermedad a través de factores de exposición comunes que moldean patrones regionales. Un ejemplo clásico de este vínculo se observa entre exposiciones ambientales y el desarrollo de padecimientos, ya sea que se trate de la exposición a residuos de actividades económicas como la minería (Manzanares Rivera, 2016) o bien de la diseminación de virus entre la población (Manzanares Rivera, 2017). No obstante, hasta ahora, esta relación territorial para afecciones comúnmente vinculadas al área de salud mental como el suicidio, tiene menor evidencia.

El análisis de la ocurrencia de suicidio desde una perspectiva territorial, por ejemplo, revela que en México se observa un alto grado de heterogeneidad con entidades en donde de hecho el fenómeno ha decrecido, como el caso de la Zona Metropolitana de la ciudad de México, con una población que supera los 20 millones de habitantes (CONAPO, 2010) donde se observó un descenso del 28,5\% para la población de sexo masculino, al pasar de siete individuos a solo cinco por cada 100 mil habitantes y del $50 \%$ entre la población femenina, al pasar de dos a una en las últimas dos décadas ${ }^{1}$.

1 Importante destacar que si bien este comportamiento está influenciado por la dinámica demográfica en el 
Mientras, se tienen entidades como Aguascalientes, en el centro norte del país que han transitado hacia causas de mortalidad prevenibles que no figuraban hace dos décadas. Dicho estado enfrenta actualmente un problema de incidencia crítica con el mayor crecimiento de suicidio como causa de muerte en el país. Aguascalientes ha registrado un incremento ajustado por estructura de edad de un orden en magnitud de cinco a uno para la población masculina, al pasar de tres defunciones por cada 100 mil habitantes a 18 entre 1998 y 2017 y para la población femenina un crecimiento que pasó de cero casos en 1998 a tres en 2017.

Paradójicamente, el estado de Aguascalientes puede considerarse como un caso de éxito en términos de crecimiento económico ${ }^{2}$, con indicadores macroeconómicos que duplican de manera sistemática los observados a escala nacional, un hecho que a priori, supondría una mejoría de los estándares de vida de su población. Si bien esta correlación encuentra profundas raíces, desde las ideas de clásicos como Adam Smith (citado en Bunge, 2012), otros autores caracterizan el crecimiento económico como un proceso, en el que de hecho, se generan brechas que implican la concentración de los recursos en unos cuantos, generando efectos negativos que afectan la calidad de vida de los individuos y el bienestar de las comunidades (Douthwaite, 2011, p 96); la "ilusión del crecimiento", como la llama Douthwaite, en última instancia supone una relación opuesta a la visión idealista de clásicos como Smith y otros autores contemporáneos en donde el crecimiento económico se asocia generalmente con un incremento en la calidad de vida, particularmente con aspectos como la salud (Cutler, Deaton y Lleras-Muney, 2006) y el bienestar de los individuos en una sociedad.

Para el caso de Aguascalientes, este acelerado crecimiento ha impactado el tejido social a través de cambios estructurales principalmente, con una activa corriente migratoria que impulsó la expansión del sector inmobiliario en búsqueda por satisfacer la demanda por vivienda generada por este proceso demográfico. Según sugieren investigaciones pioneras para el caso de Aguascalientes, la adaptación a este nuevo entorno económico ha resultado en efectos sociales adversos, tales como el aumento de la polarización, incremento de los índices de criminalidad y el hacinamiento (Enriquez-Aranda, 1995; Franco Muñoz et al., 2004), hipótesis que ha suscitado el interés de la comunidad científica (Hermosillo, Vacio, Ponce de León, Ortega y Macías, 2015), que reconoce la necesidad de un abordaje multidisciplinario.

A pesar de la evidencia a escala global que vincula determinantes de índole económico como las condiciones de vivienda con impactos en salud mental (Marí-Dell'Olmo et al., 2017), el abordaje de este fenómeno, considerando su distribución espacial y evolución temporal, es aún un enfoque explorado escasamente para el caso de México (Borges, Orozco, Benjet y Medina-Mora, 2010; Martínez Salgado, 2010).

La existencia de fuentes de información que aportan un gran volumen de datos, como son los registros electrónicos de mortalidad, representan una fuente valiosa para estudiar el tema, a pesar de los retos inherentes al registro del fenómeno, incluido el subregistro debido al estigma social (Sánchez-Cervantes, Serrano-González y Márquez-Caraveo,

estado, la magnitud de la reducción en la causa suicidio sugiere que se trata de un decremento efectivo, ya que la magnitud de la reducción en el estado fue de $6,2 \%$ en la tasa de mortalidad entre la población masculina y del $20 \%$ entre la población femenina.

2 La entidad alcanzó un ritmo de crecimiento promedio anual del Producto Interno Bruto (PIB) de 4,23\% durante la década, un desempeño que prácticamente duplicó el ritmo nacional que fue 2,3\%, y en 2016 este indicador macroeconómico registró una variación anual de 9,8\%; el mayor crecimiento en México. 
2015), y al entono cultural de cada país (Hernández-Bringas y Flores-Arenales, 2011).

En este trabajo el objetivo es estudiar la evolución del fenómeno de suicidio en México, desde una perspectiva territorial a escala estatal durante el periodo 1998-2017 y documentar las diferencias en las tasas de mortalidad con énfasis en el caso del estado de Aguascalientes, que presenta la mayor tasa de mortalidad en el país. El periodo seleccionado cubre la totalidad de registros públicos disponibles a la fecha a través del Sistema Nacional de Información en Salud.

Adicionalmente se aplica por primera vez en México para el análisis de suicidio la herramienta de Google trends para detectar posibles patrones de estacionalidad y validar la relación entre tasas de suicidio y patrones territoriales de búsqueda en internet para el término suicidio.

\section{ESTUDIOS PREVIOS Y CONSIDERACIONES TEÓRICAS}

La investigación se conceptualiza desde la perspectiva teórica de los determinantes sociales de salud, en esta propuesta teórica, el conocer los patrones geográficos y su evolución en el tiempo permite aproximar el estudio de las causas, lo que es útil para perfilar estrategias de monitoreo basadas en evidencia. Al respecto la Organización Mundial de la Salud (OMS) recientemente ha fomentado una serie de iniciativas centradas en la aplicación de este paradigma, enfatizando su vínculo hacia el diseño de políticas públicas (WHO, 2017a).

El planteamiento del análisis representa una crítica a la aproximación de suicidio centrado en el individualismo clínico, al respecto autores pioneros en este campo como Shneidman aseveran que: "La clave de la prevención del suicidio yace en enfocarse en el dolor psicológico idiosincrático del individuo" (Pompili, 2018, p. vii) (traducción nuestra). Afirmación que expresa el papel que el enfoque individual en el estudio de este fenómeno tradicionalmente ha tenido.

Develar patrones propios del contexto como la evolución temporal o la distribución espacial, se considera pertinente ya que permite aportar rasgos explicativos complementarios a los estudios realizados desde una perspectiva individual, que ya han documentado, con cierto grado de consenso, los factores de riesgo clínicos asociados a este fenómeno (García-Rábago et al., 2010; Meyer, Irani, Hermes y Yung, 2017; Rizvi, Iskric, Calati y Courtet, 2017; Shneidman, 1993).

En términos teóricos, el trabajo seminal de Durkheim (1951), que abordó entre otros elementos, el nexo entre las emociones, en tanto puente entre las decisiones que los individuos toman y el entorno social más amplio, en el que ellos se ubican, constituye un antecedente clásico. De hecho, Durkheim consideró las variaciones geográficas y estacionales observadas en el fenómeno del suicidio, para enfatizar el papel de los determinantes sociales; "necesariamente debe depender de causas sociales y ser en sí mismo un fenómeno colectivo... las variaciones geográficas y estacionales del suicidio, definitivamente nos han conducido a esta conclusión" (Durkheim, 1951, p. 97) (traducción nuestra). Es precisamente este mecanismo entre factores exteriores al individuo y el desarrollo de afecciones de salud mental, una vertiente que ha cobrado auge dada la evidencia empírica reportada por estudios recientes.

Así por ejemplo, el análisis del flujo masivo de información generado por: dispositivos móviles, el uso de redes sociales como Twitter o motores de búsqueda como Google, ha demostrado un potencial para revelar diversas expresiones de sentimiento por parte de los 
individuos, tal como lo reportan Bruns, Dunkel y Offel (2019) entre otros. Y permite también revelar patrones a diferentes escalas de desagregación geográfica (Howard, Woolley y Ryan, 2018; Keller y Ulrike, 2019), por lo que constituye una perspectiva que es de interés para contribuir en la caracterización de fenómenos de salud pública como el suicido.

En el plano empírico, una hipótesis relevante sobre todo por su aplicación para países de ingresos medios y bajos se refiere al impacto que condiciones sociales estructurales como la pobreza tienen respecto a los índices de suicidio. Estudios realizados por lemmi et al. (2016) demuestran la importancia de estos factores estructurales. A pesar de que se reconoce que las condiciones de pobreza por sí mismas no son un determinante causal directo, la evidencia reportada en este sentido indica que la población en situación de pobreza esta expuesta a factores que inciden en manifestaciones de disparidades de salud, incluidas aquellas afecciones de salud mental (Bonnefoy, 2007; Evans, Wells y Moch, 2003). Algunos factores documentados incluyen: habitar en viviendas que presentan condiciones de infraestructura menos favorables (Aguilar y López, 2016; Rojas, Meichtry, Ciuffolini, Vázquez y Castillo, 2008), tales como: menores dimensiones con mayores problemas de hacinamiento (Jusidman, 2012; Ortiz, 2017), o localizadas en zonas periféricas (Libertun de Duren, 2018; Rasse, 2015).

Específicamente la relación entre condiciones de vivienda como el hacinamiento y problemas de salud pública como el suicidio, ha generado particular interés en áreas urbanas de países en desarrollo en la región de América Latina como Argentina (Leveau, Guevel y Alazraqui, 2019); Brasil (Bando, Moreira, Pereira y Barrozo, 2012); México (Hermosillo et al., 2015); y Chile (Urria-Yánez, 2018). Esta relación se ha abordado también para diversos grupos poblacionales, incluida la población indígena, como lo demuestran Pepin, Muckle, Moisan, Forget-Dubois y Riva (2018).

Sin embargo, se observa que la dirección y la magnitud de la relación depende de la definición del indicador de hacinamiento estudiado, tal como lo documentan Leveau et al. (2019), quienes, al incluir esta dimensión en la conformación de un índice de pobreza para un estudio de caso en Argentina, encuentran que la relación no es estadísticamente significativa.

En contraste, la Organización Mundial de la salud (OMS) a través de sus recomendaciones guía sobre condiciones de vivienda, advierte que la situación de hacinamiento puede tener efectos adversos sobre condiciones de salud mental y se asocia con situaciones de mayor estrés (WHO, 2018), aunque no específicamente con un mayor riesgo de suicidio; por lo que el consenso sobre el papel que la condición de hacinamiento tiene para el fenómeno de suicidio, es una vertiente de estudio aún en desarrollo.

En términos empíricos, y mientras la investigación en esa línea en otras regiones del mundo ha permitido la orientación de políticas públicas sobre los proyectos de construcción de vivienda (Marí-Dell'Olmo et al., 2017), desde la perspectiva regional en América Latina, autores como Rufino (2015) argumentan que la política de vivienda reciente ha reforzado y ampliado los procesos de segregación espacial.

En particular para el caso de México, Montejano Escamilla, Caudillo Cos y Cervantes Salas (2018), documentan que durante el primer decenio del siglo XXI, la política de desarrollo de vivienda social en México promovió la construcción masiva de nuevos conjuntos habitacionales en municipios de las periferias urbanas y favoreció una baja mezcla de uso de suelo. Evidencia que concuerda con los estudios previos de Eibenschutz y Goya (2009) quienes advierten sobre los problemas de accesibilidad generados para atributos como los centros de empleo, equipamiento educativo, salud, cultura y recreación. Con- 
ceptualmente la integración de los elementos anteriores, también se ha desarrollado bajo la noción de ciudades saludables (Cole et al., 2017), un paradigma que enfatiza las interrelaciones sociales con los factores de riesgo individuales. Así, una hipótesis central en esta perspectiva conceptual es que el entorno social y en particular el económico puede impactar en las dimensiones de salud como el desarrollo sicológico de las personas, una dimensión fundamental para comprender la ocurrencia del suicidio (Schlimme, 2018).

\section{DATOS Y MÉTODOS}

En primer lugar, se realiza un análisis exploratorio de datos (EDA), utilizando los registros de mortalidad recopilados por el Sistema Nacional de Información de Salud (SINAIS). Estos registros cumplen con protocolos en materia de estandarización de información en salud como la Norma Oficial Mexicana 035-SSA3-2012, lo que implica que los mismos procesos y definiciones de registro se aplican en todo el país (Diario Oficial de la Federación, 2012b). Para lograr resultados comparables en el contexto internacional, esta fuente de datos se basa en la clasificación internacional de enfermedades CIE-10.

Para efectos de este trabajo el suicidio se define como la muerte ocasionada por lesión auto infligida informada como intencional. Se consideran las causas de muerte en el rango de códigos (X60-X849), rango que incluye información específica sobre el deceso y permite desagregación a escala geográfica, así como una disponibilidad anual desde 1998. Con la finalidad de capturar la evolución temporal de este fenómeno de salud pública, se utilizan series de tiempo para la totalidad de información oficial disponible hasta el momento en el Sistema Nacional de Información en Salud con un total de 20 años en el periodo 1998-2017.

Si bien la estructura conceptual de los registros de mortalidad mantiene un componente geográfico homogéneo a lo largo de todos los años de la serie con dos escalas de registro básicas que son: el estado y el municipio, a partir del año 2012; el SINAIS estableció ajustes regulatorios definidos por la norma Oficial mexicana NOM-024SSA3-2010 para mejorar la interoperabilidad, confidencialidad, seguridad y uso de estándares en la captura de la información de los registros electrónicos en salud (Diario Oficial de la Federación, 2012a). Esta regulación implicó la inclusión de cambios conceptuales que permiten atender entre otros temas la diversidad de pueblos indígenas presentes en México, como es el caso de la variable que indica si la persona habla lengua indígena y una variable geográfica que distingue el lugar de registro del hecho. Lo que complementa la información ya existente sobre el lugar de la defunción y la residencia habitual. En el caso particular, el análisis se basa en el sitio de residencia habitual. Adicionalmente, acorde a las convenciones internacionales sobre la protección a los datos personales, las bases no incluyen datos que permitan identificar a los pacientes de manera puntual.

En la primera fase del análisis se presenta el escenario base de este fenómeno, mediante la estimación de las tasas de mortalidad ajustadas por estructura de edad y sexo para el año más reciente de la muestra (2017) aplicando el método directo con la población estándar proveniente de las proyecciones de población a 2017. De forma complementaria se estiman las tasas de crecimiento de los casos por suicidio respecto a otras causas de muerte para las 32 entidades del país, así como el comportamiento desagregado para las principales causas específicas para el estado de Aguascalientes, así como la distribución de la frecuencia según el mes de ocurrencia del deceso. 
Las tasas de crecimiento para las series de tiempo se estiman con relación al año base, en este caso 1998. Para obtener el crecimiento en términos porcentuales, la serie se expresa mediante su trasformación logarítmica. Para garantizar la comparabilidad tanto intra estado como entre los 32 estados del país, se incluye el comportamiento de una segunda serie que captura la trayectoria de la mortalidad de todas las causas excepto el suicidio. En ambas series la estructura poblacional para un año particular es la misma, por lo que el contraste entre un año y otro para un mismo estado es factible. Por su parte, la magnitud de la brecha que ya controla por la estructura poblacional, aporta un indicador comparable entre los estados.

El grado de concentración geográfica de muertes por suicidio se determina utilizando como unidad de análisis la Zona Metropolitana (ZM). Las zonas metropolitanas como espacios de análisis presentan ventajas en términos empíricos ya que se trata de espacios de menor dimensión que la escala estatal. De hecho, las zonas metropolitanas se integran por un sub conjunto de municipios que mantienen una relación funcional entre sí (CONAPO, 2010), lo que puede ser útil para capturar el comportamiento regional de procesos demográficos en el dominio de salud pública.

Por definición las ZM incorporan una relación funcional entre los espacios geográficos que las componen con una ciudad central, que dados sus atributos de localización resultan polos de atracción y fungen como articuladoras de la actividad. En México se tienen identificadas 59 zonas metropolitanas ${ }^{3}$, incluida la Zona Metropolitana de Aguascalientes, que se integra por tres municipios a partir de la ciudad de Aguascalientes como el centro de actividad.

Considerando esta unidad de análisis se lleva a cabo la estimación del cociente de localización LQ, un indicador que ha sido aplicado por estudios en el campo de la bioestadística y en estudios empíricos de corte epidemiológico dado su potencial para detectar patrones de comportamiento espacial, ya que permite medir la concentración relativa de casos controlando por el tamaño de la población (Djira, Schaarschmidt y Fayissa, 2010). La estimación de cociente de localización (LQ) se realiza mediante la siguiente especificación:

$$
\mathrm{LQ}_{t}=\begin{aligned}
& C / T_{j} \\
& C / T_{N}
\end{aligned}
$$

Donde el numerador indica la proporción de casos por suicidio respecto al total de defunciones en el espacio geográfico $j$, mientras el denominador captura la proporción correspondiente en el ámbito nacional, ambas proporciones se estiman para un periodo de tiempo concreto $t$.

Por definición, el umbral $\mathrm{LQ}=1$ indica una situación en la que la proporción por defunciones observada, tanto en el sitio $j$ como en la escala nacional (ámbito de referencia), es la misma; mientras valores LQ >1 indican una situación de concentración relativa superior en el área $j$ relativa al contexto nacional y por lo tanto constituyen áreas de interés para efectos de esta investigación. Adicionalmente para mostrar el contraste de este fenómeno respecto a otras causas de muerte e identificar grupos etarios con mayor vulnerabilidad, se construyen curvas de densidad para la edad del fallecimiento.

3 En 2018 el listado de zonas metropolitanas se actualizó a 79, sin embargo, en la presente investigación se consideran las 59 ZM diseñadas con base en el censo 2010 y no la información de la Encuesta Intercensal 2015, un instrumento con un nivel de representatividad menor. 
En la segunda fase del análisis, el enfoque metodológico se basa en la utilización del motor de búsqueda Google trends que captura las preferencias de los usuarios. El propósito es examinar la relación de las tasas de suicidio y el score que captura la importancia relativa del término 'suicidio' durante los últimos cinco años a escala estatal. Esta herramienta ha sido empleada en estudios empíricos diversos como los de Higgins et al. (2020); Soreni, Cameron, Streiner, Rowa y McCabe (2019); Chandler (2018); Ma-KeIlams, Or, Baek y Kawachi (2016); Mok, Jorm y Pirkis (2015), y se aplica por primera vez en México para el análisis de suicidio.

Este score refleja el interés de búsqueda en relación con el valor máximo en un espacio geográfico y un periodo determinados. Un valor de 100 indica la popularidad minviernoáxima de un término, mientras que 50 y 0 indican que un término es la mitad de popular en relación con el valor máximo, o que no había suficientes datos del término, respectivamente (Google, 2018). Una limitación de esta fuente de datos es que el perfil de la persona que realiza la búsqueda no es un dato público, lo cual se basa en las políticas de protección a la privacidad de los usuarios definidas por Google. A pesar de esta limitante, esta fuente de datos aporta dos dimensiones de utilidad, que son la espacial con escala país, estado y ciudad; y la información temporal, lo que permite explorar las tendencias para momentos de tiempo predefinidos.

Con este insumo se estimó un modelo de regresión lineal simple ${ }^{4}$ que toma como variable dependiente la tasa observada de suicidio por estado y como variable independiente el score de Google trends para el término 'suicidio'. El propósito de esta aplicación metodológica es aportar información de contraste que complementa los resultados obtenidos con los indicadores tradicionales como las tasas de mortalidad ajustadas y aprovechar la disponibilidad de grandes volúmenes de datos que los medios masivos de comunicación como internet, generan. Importante mencionar que el score generado por la herramienta Google trends considera las preferencias reveladas de los individuos y como tal, permite clasificar las tendencias de búsqueda a escala geográfica, por lo que no se trata de un indicador de causalidad para el suicidio. Este refleja el interés de los usuarios de la plataforma por un término especifico en un momento de tiempo determinado, es un indicador de la popularidad de un término.

El indicador se encuentra normalizado, lo que implica que cada uno de los datos se divide por el total de búsquedas del término para el territorio geográfico de interés y el periodo de tiempo determinado. ¿Hasta qué grado este indicador observa correlación con las tasas de mortalidad estimadas a partir de los registros del sistema nacional de información en salud? Es una pregunta que requiere validación empírica formal y que se examina como parte del presente estudio.

\section{RESULTADOS}

INDICADORES DE CONTEXTO

En primer lugar se estiman las tasas de mortalidad por suicidio ajustadas por edad y sexo, un indicador estándar en la literatura que permite contar con un escenario base

4 Para confirmar el cumplimiento de los supuestos clásicos del modelo de regresión y la validez de los estimados, se aplicaron las pruebas de Breusch-Pagan para detectar posibles problemas de heteroscedasticidad. Durbin-Watson para detectar posibles problemas de autocorrelación en los residuales y se validó el supuesto de normalidad en los residuales aplicando la prueba de Shapiro-Wilk. Dado que se trata de un modelo de regresión simple, no se aplican pruebas para examinar multicolinealidad. 
de contraste. Se realiza la estimación para las 32 entidades federativas del país. La Figura 1 muestra el resultado.

Figura 1. Tasas de mortalidad por suicidio ajustadas por edad y sexo según entidad federativa. México, 2017

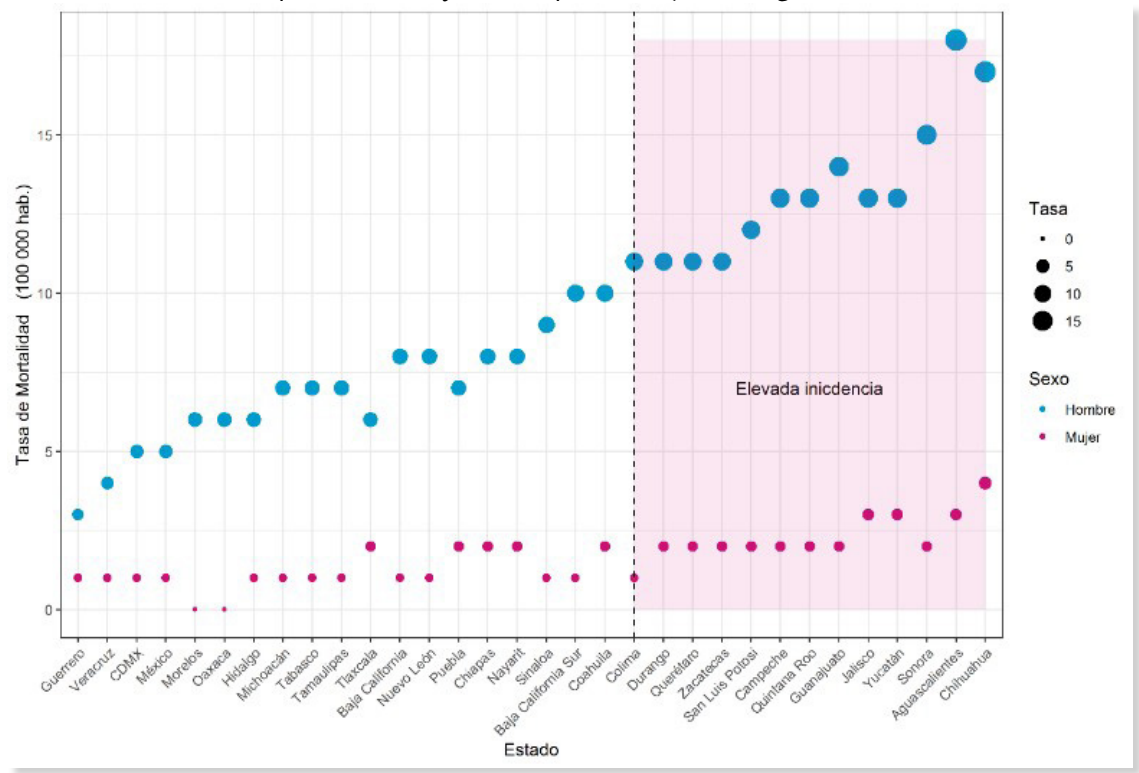

Fuente: elaboración personal con base en datos de SINAIS (2017).

Proyecciones de población Consejo Nacional de Población. CONAPO

La estimación revela un claro patrón de mayor incidencia para el sexo masculino entre los estados que integran el país, este alcanza un valor máximo en el estado de Aguascalientes con 18 personas por cada 100.000 habitantes. En el caso del sexo femenino, la mayor magnitud se registra en el norte del país, en el estado fronterizo de Chihuahua, con cinco mujeres por cada 100.000 habitantes. A escala de país se observa que la tasa promedio para el sexo masculino cuadruplica la estimada para el sexo femenino.

Para facilitar la interpretación se incluye una línea de referencia que permite identificar un subconjunto de entidades consideradas de elevada incidencia cuyas tasas estimadas superan la tasa de mortalidad promedio nacional. Este subconjunto incluye los estados de Aguascalientes, Chihuahua, Yucatán, Campeche y Sonora como los de mayores tasas. Asimismo, se observa que entidades de importancia por la magnitud en su dinámica demográfica como la ciudad de México (CDMX) que alberga el principal centro de actividad económica y la mayor concentración poblacional del país, de hecho, se ubican dentro del subconjunto inferior de la distribución con una tasa de cinco hombres y una mujer. La estimación revela adicionalmente que los estados de Guerrero, Veracruz y CDMX presentan los menores niveles de mortalidad a causa de suicidio en México.

A continuación se muestra la evolución temporal del suicidio como causa de muerte para las 32 entidades del país. Este cálculo permite distinguir aquellas entidades en las que se observa un problema persistente de largo plazo, así como para identificar los estados en donde el crecimiento da cuenta de un fenómeno de reciente aparición. La Figura 2 muestra los resultados.

Si bien la información sobre tasas de mortalidad permite contar con un escenario base de partida sobre la situación actual, la construcción de la serie temporal que se presenta en la Figura 2, aporta rasgos detallados sobre la evolución de este fenómeno que nos permite profundizar en su entendimiento. Se encuentra que el estado de Aguas- 
calientes destaca por el comportamiento sistemático y la magnitud del crecimiento a través de toda la serie. De hecho, muestra un crecimiento en casos de suicidio entre 1998 y 2017 de 194,5\%, la trayectoria de mayor crecimiento en el país, lo que se traduce en un ritmo de crecimiento anual de $9,7 \%$; un avance que cuadruplica el observado por el resto de casusas de muerte que es de solo $2,3 \%$ anual.

Figura 2. Tasa de crecimiento de defunciones por suicidio según entidad federativa Mexico.1998-2017

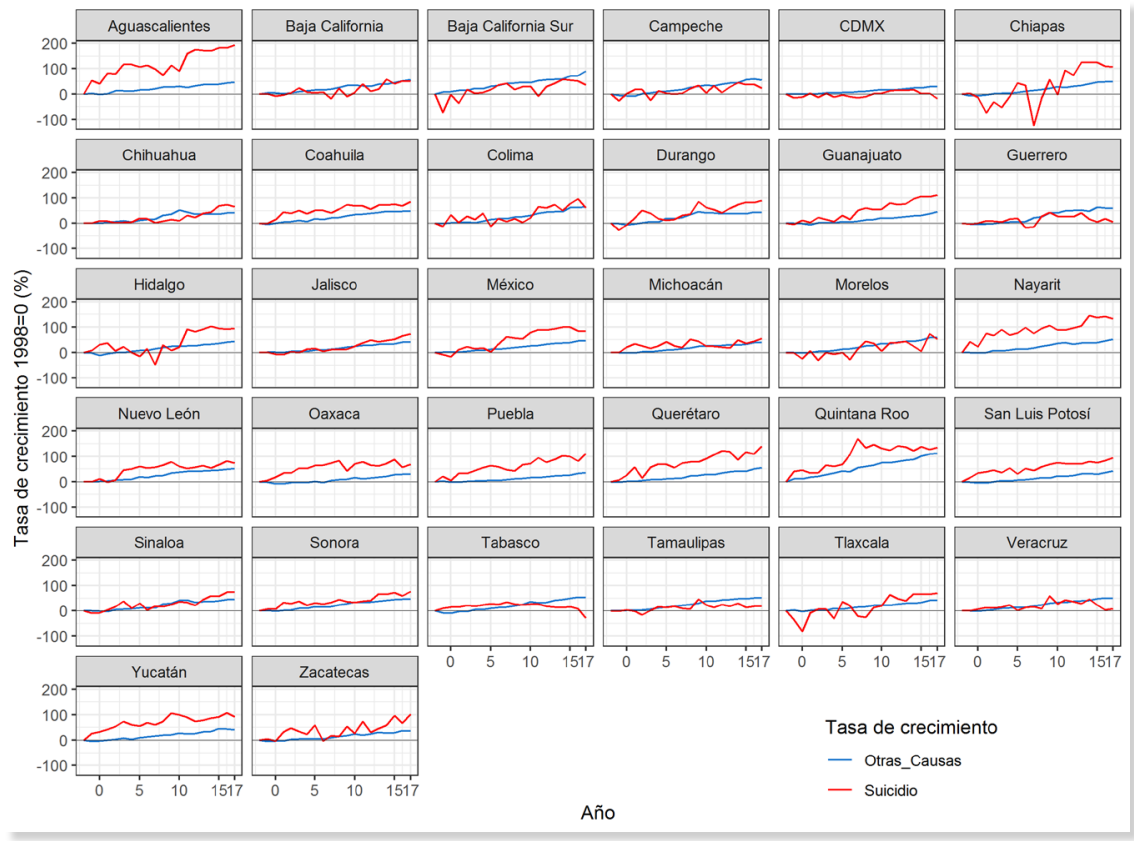

Nota: Se utiliza una escala logaritmica normalizada con año base 1998

Fuente: elaboración personal con base en datos de SINAIS, Secretaría de salud (1998-2017)

Adicionalmente la magnitud de la evolución a lo largo del periodo de estudio resulta evidente al contrastar con el comportamiento de un conjunto de entidades como CDMX o Tabasco donde se observa una reducción de esta causa de muerte equivalente al 19\% y $28 \%$ respectivamente ${ }^{5}$.

Durante este periodo, la tasa de mortalidad por suicidio para el sexo masculino en Aguascalientes pasó de ubicarse de la posición 27, a ocupar la primera posición entre los 32 estados del país, con un ascenso en su tasa de mortalidad de tres hombres y cero mujeres por cada 100.000 habitantes a 18 y 3 respectivamente. Un segundo elemento de análisis se expresa por la magnitud de la brecha alcanzada entre la trayectoria de los casos de muerte por suicidio y el resto de las causas de muerte. Esta representa un insumo de interés sobre la importancia relativa de este problema en cada entidad y permite controlar el posible sesgo inducido por su dinámica demográfica, en particular el impacto del componente migratorio. Lo anterior debido a que desde la perspectiva conceptual tanto la tasa de mortalidad causa especifica como la tasa de mortalidad general comparten la misma estructura poblacional. Nuevamente el estado de Aguascalientes exhibe una brecha de crecimiento respecto al resto de causas de muerte equivalente a $147,6 \%$, por lo que la evolución observada en este caso es consistente con un problema de largo plazo.

5 Las entidades de CDMX, Tabasco y Guerrero registraron el menor crecimiento del suicidio como causa de muerte en todo el país en el periodo estudiado. 
Además de Aguascalientes, en el grupo de mayor brecha de crecimiento ${ }^{6}$ respecto al resto de causas figuran los estados de Querétaro, Nayarit, Puebla, con un diferencial de crecimiento de $84 \%, 81 \%$ y $75 \%$ respectivamente ${ }^{7}$.

La escala de análisis a partir del comportamiento estatal aporta rasgos distintivos sobre la manifestación del suicidio en México. Sin embargo, con la finalidad de obtener un mayor detalle de la concentración relativa de este fenómeno, se analiza su comportamiento para las 59 zonas metropolitanas existentes en México. Esta escala de análisis con mayor desagregación, permite identificar aquellos centros poblacionales donde se concentra la ocurrencia de este fenómeno con mayor precisión.

Se estima a continuación el cociente de localización (LQ), un indicador de concentración relativa que permite controlar por el tamaño poblacional en cada unidad de análisis. La estimación se hace primero a escala estatal para los 32 estados que integran el país y para los 20 años de información disponible. En una segunda fase, se estima el indicador de concentración para las 59 zonas metropolitanas, una escala geográfica que aporta un mayor detalle. Los resultados se presentan en la Figura 3a y 3b; mientras que la Figura 4 muestra el mapa de referencia.

Figura 3a. Cociente de localización (LQ) anual a escala Estatal México de 1998 a 2017 AG BC BS CA CD CH CL CO CS DG GR GT HG JA MI MO MX NA NL OA PU QR RO SI SL SO TA TL TM VR YU ZA 6

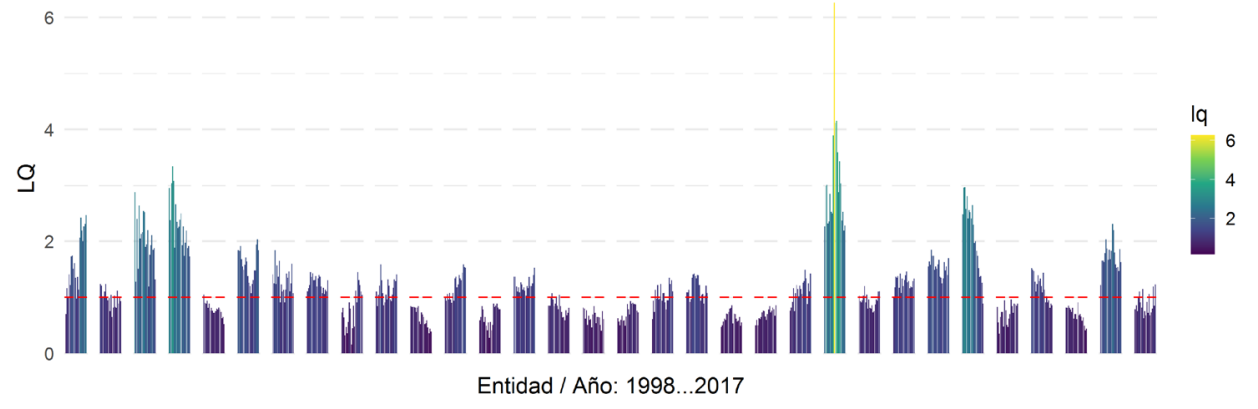

Nota: la línea roja de referencia muestra el nivel de contraste (concentración en el país) Fuente: elaboración personal con base en datos de SINAIS, Secretaría de salud (19982017). Proyecciones de población Consejo Nacional de Población (CONAPO)

El patrón encontrado permite confirmar los hallazgos de la tasa de mortalidad, un indicador clásico de referencia. Sin embargo, ahora podemos notar que además de Aguascalientes y Quintana Roo tenemos otros estados en los que la causa de muerte por suicidio ha presentado una manifestación persistente en la últimas dos décadas no obstante con una clara tendencia decreciente, los casos de Tabasco y Campeche en la frontera sur del país o Tamaulipas y Baja California Sur en la frontera Norte son representativos de este fenómeno.

La Figura 3b muestra la estimación a escala de Zona Metropolitana para el año más reciente disponible en los registros de mortalidad del sistema nacional de información en salud. Una escala pertinente en cuanto a que captura la importancia relativa de los principales centros poblacionales al interior de cada estado.

6 El grupo de mayor brecha de crecimiento se define a partir del ordenamiento ascendente de la brecha estimada, considerando los cinco estados de mayor magnitud en este indicador.

7 Es necesario puntualizar sobre la inducción de sesgo derivado de la dinámica demográfica en cada entidad, particularmente debido al componente migratorio, un factor con influencia mayor en los casos de Quinta Roo y Querétaro. No obstante para controlar este posible sesgo se calculó la brecha respecto a la trayectoria de crecimiento del resto de causas de muerte, lo que permite aislar el comportamiento diferencial respecto al suicido. 
Figura 3b. Cociente de localización (LQ) por Zona Metropolitana (ZM) México 2017

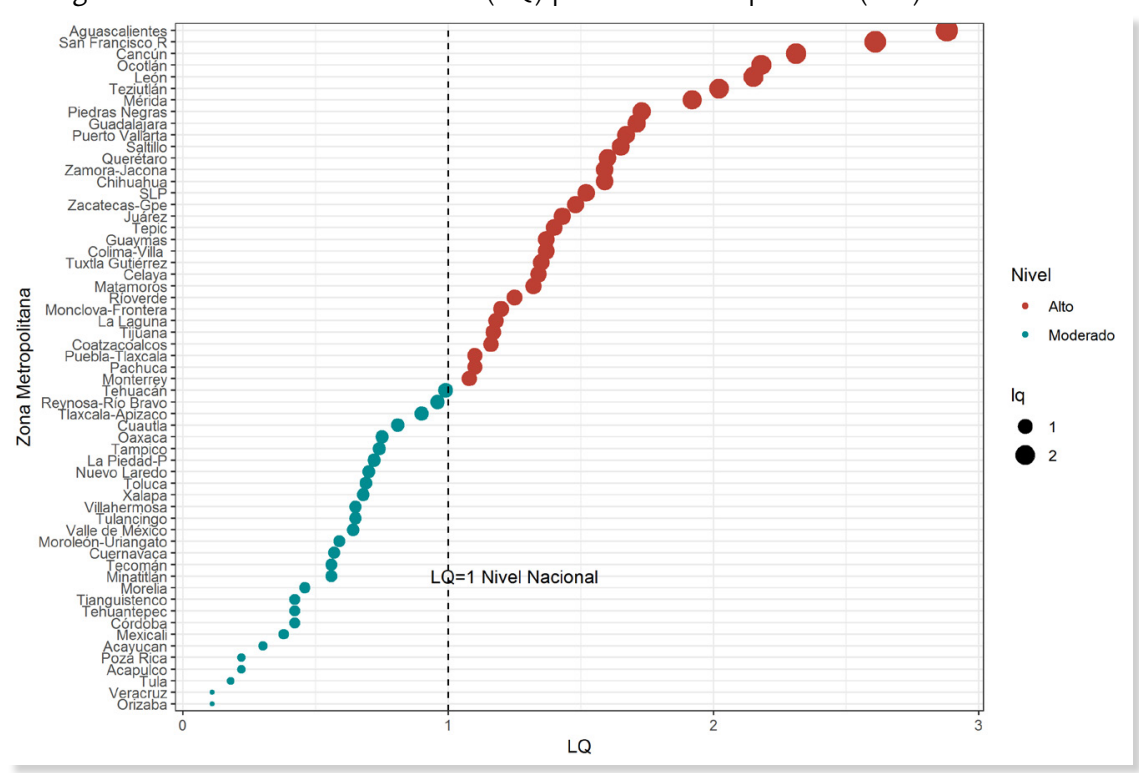

Fuente: elaboración personal con base en datos de SINAIS (2017). Proyecciones de población Consejo Nacional de Población (CONAPO)

Los resultados permiten obtener elementos de análisis a una escala con mayor desagregación que complementan el patrón revelado a escala estatal, así se encuentra que la Zona Metropolitana de Aguascalientes presenta el mayor cociente de localización ( $L Q=2.88$ ), un nivel que duplica el escenario observado en el país, lo que confirma la severidad del problema revelado por la trayectoria temporal de crecimiento y las tasas de mortalidad. Adicionalmente zonas metropolitanas como Cancún en el estado de Quintana $\operatorname{Roo}^{8}$ y San Francisco del Rincón en el estado de Guanajuato registran un problema acentuado de muertes por suicidio con un $L Q=2.31$ y 2.61 respectivamente.

Adicionalmente la estimación anterior permite crear una clasificación integrada por aquellas zonas metropolitanas de alta incidencia, cuyo nivel de concentración supera el escenario nacional y distinguir aquellas donde el suicidio ocurre con una concentración relativa menor. Dentro del primer grupo se define un subconjunto de zonas metropolitanas denominadas de incidencia crítica que se integra por Cancún, Quintana Roo, Aguascalientes, Aguascalientes, Puerto Vallarta, Jalisco, Chihuahua, Chihuahua y la Zona Metropolitana de León en el estado de Guanajuato9 (Figura 4).

Se observa que los resultados del indicador relativo de concentración LQ, son consistentes con el escenario delineado por los indicadores previos, asimismo la escala de ZM muestra con mayor precisión la problemática que se tiene en áreas de intenso crecimiento económico como Aguascalientes, Chihuahua, Cancún, Puerto Vallarta o León; zonas metropolitanas en las que se encuentran interesantes rasgos compartidos, ya que se trata de urbes que han experimentado un dinámico crecimiento económico en las últimas décadas. En los dos primeros casos con un crecimiento económico fuertemente

8 La ciudad de Cancún es notoria ya que es el principal destino turístico del estado de Quintana Roo y se ha convertido en un importante polo de desarrollo económico acompañado por un importante flujo migratorio.

9 Este subconjunto considera como umbral el límite superior del rango intercuartil para la distribución del cociente de localización estimado en las 32 entidades. Este valor corresponde a $L Q=1.37$. De este modo, las entidades que integran el subconjunto de incidencia crítica presentan valores de concentración LQ>1.37. 
asociado al sector manufacturero de exportación mientras en los casos de Cancún y Puerto Vallarta ambos notables polos de crecimiento a partir del sector turismo.

La Figura 5 muestra la evolución de casos distinguiendo las causas de suicidio específicas, así como la distribución de la frecuencia según el mes de ocurrencia del deceso, la estimación considera el caso de Aguascalientes una entidad que, de acuerdo a los indicadores estimados, enfrenta un problema de incidencia crítica y el mayor crecimiento de suicidio como causa de muerte en el país durante el periodo 1998-2017.

Figura 4. Ubicación de zonas metropolitanas en México 2017 y Cociente de Localización (LQ)

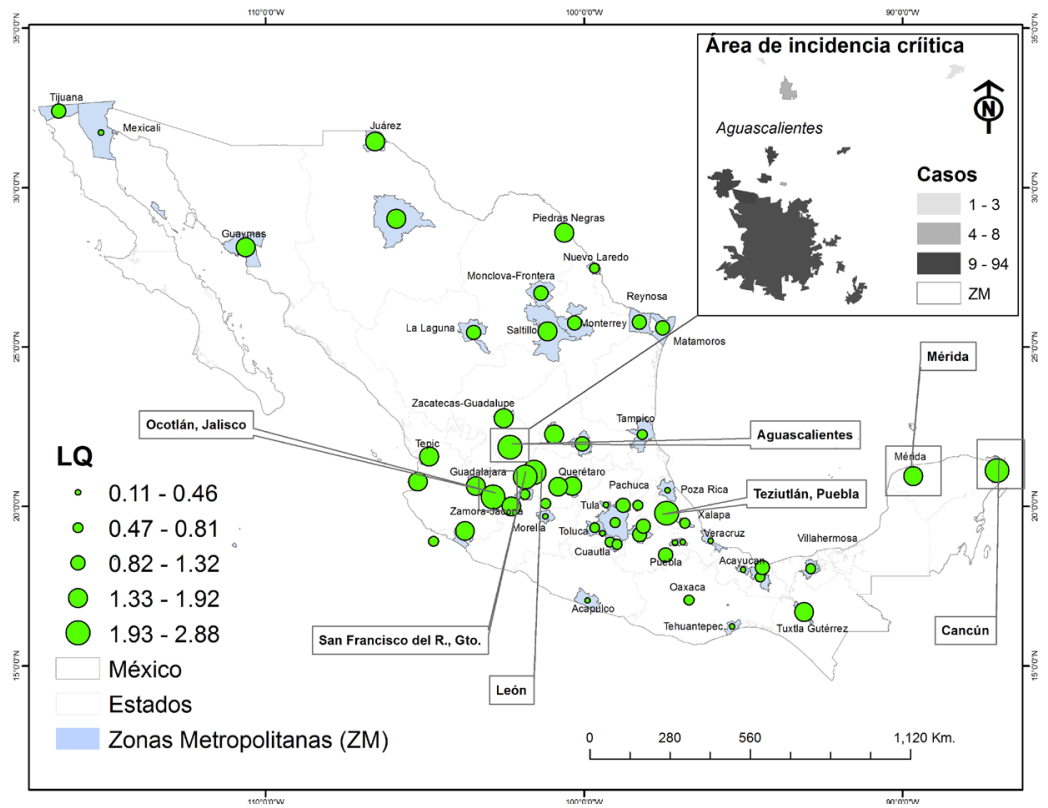

Fuente: elaboración personal con base en datos de Sistema Nacional de información en Salud (SINAIS, 2015)

Figura 5. Evolución temporal de defunciones por suicido por causa especifica. Distribución de casos por mes. Aguascalientes 1998-2017
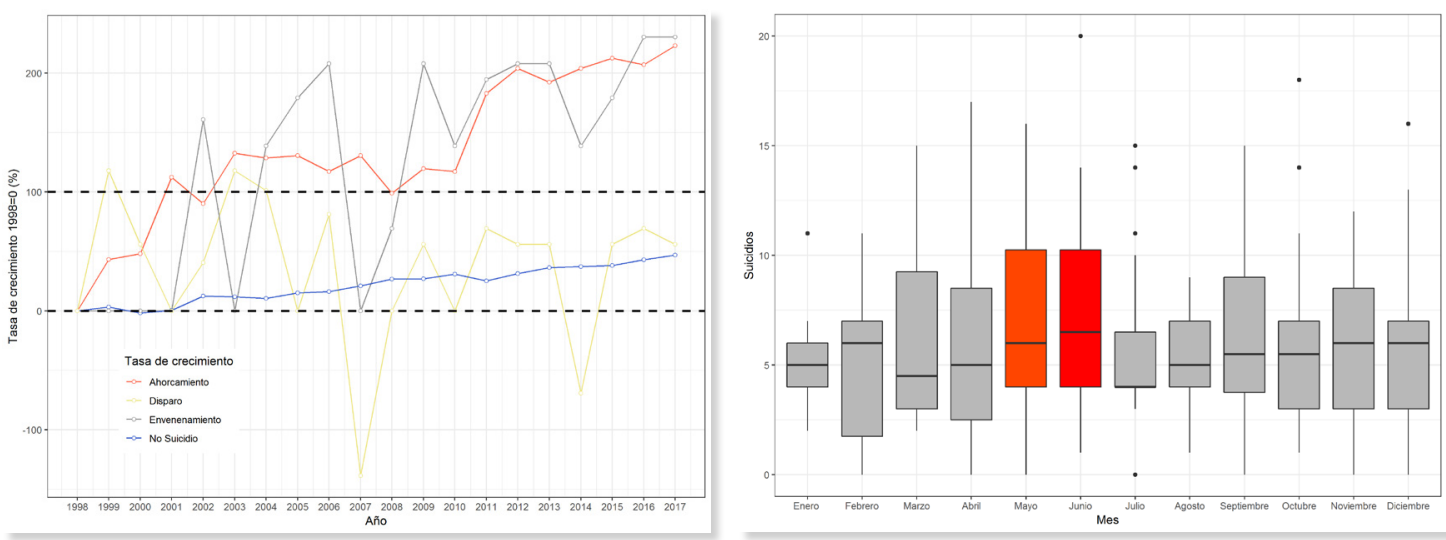

Fuente: elaboración personal con base en con datos de SINAIS (1998-2017)

Los resultados indican que la principal causa de muerte es ahorcamiento (X70-X709) seguido de envenenamiento (X60-X699) y disparo de arma de fuego (X720-X749). A pesar de las fluctuaciones temporales propias de este fenómeno, el crecimiento observado de la primera supera el $200 \%$ en el periodo de estudio en contraste al resto de causas de muerte en la entidad. Adicionalmente la evidencia muestra que el mes de mayo parece 
tener una predilección particular para la ocurrencia de este fenómeno en Aguascalientes.

Con la finalidad de ubicar los grupos etarios de mayor riesgo, en la Figura 6 se construyen curvas de densidad para la distribución por edades de fallecimiento de los casos por suicidio en Aguascalientes en el año más reciente de la muestra, se incluye la distribución correspondiente del resto de causas de muerte como medio de contraste.

Se aprecia una elevada incidencia en el rango de edad entre 12 y 32 años. La evidencia indica que el suicidio como causa de muerte es un fenómeno que ocurre a una temprana edad. Este rasgo marca una clara diferencia respecto al resto de causas de muerte ya que la distribución de edades de defunción para este periodo indica que la edad media de fallecimiento en México es de 77 años.

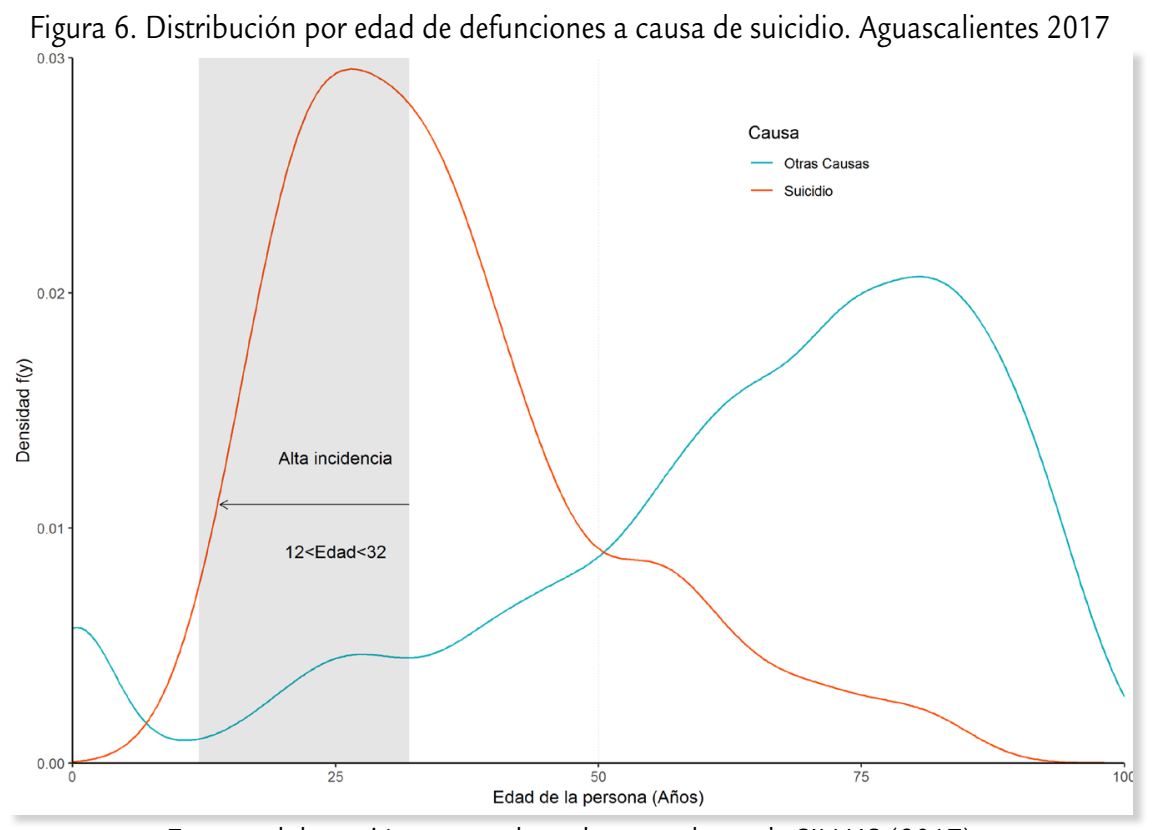

Fuente: elaboración personal con base en datos de SINAIS (2017)

\section{HaCinAMIENTO Y ÁREAS CON MAYOR INCIDENCIA DE SUICIDIO PARA EL CASO DE AGUASCALIENTES}

A continuación se aplica una escala de análisis intraurbana para representar las condiciones de hacinamiento en la zona metropolitana de Aguascalientes identificada con la mayor concentración de esta causa de muerte entre la población. Es preciso advertir que la evidencia generada por diversos autores en el contexto de áreas urbanas en países latinoamericanos permite inferir que el contexto particular del análisis, así como el uso de variables de control, tienen efecto en el nivel de significancia de la relación entre hacinamiento y suicidio. El estudio de caso realizado por Araya, Lewis, Rojas y Fritsch (2003) con datos de corte transversal, demuestra que bajo ciertas circunstancias, áreas residenciales de alta densidad con problemas de hacinamiento se vinculan con una mayor prevalencia de afecciones mentales.

Por otro lado, la complejidad de la relación entre suicidio y condiciones de vivienda, queda de manifiesto en el trabajo realizado por Leveau et al. (2019) sobre los diferenciales intra-urbanos del suicidio en Argentina, donde el efecto real de la variable hacinamiento se diluye como parte del cálculo de índices empleados principalmente para medir el estatus socioeconómico y la noción, aún más general, del fenómeno de la pobreza. 
La Figura 7 presenta la distribución de la población en hacinamiento como porcentaje de la población total por área geoestadística básica en la ciudad, en conjunto con las áreas catalogadas como de alta incidencia de suicidio por estudios previos (Hermosillo et al., 2015).

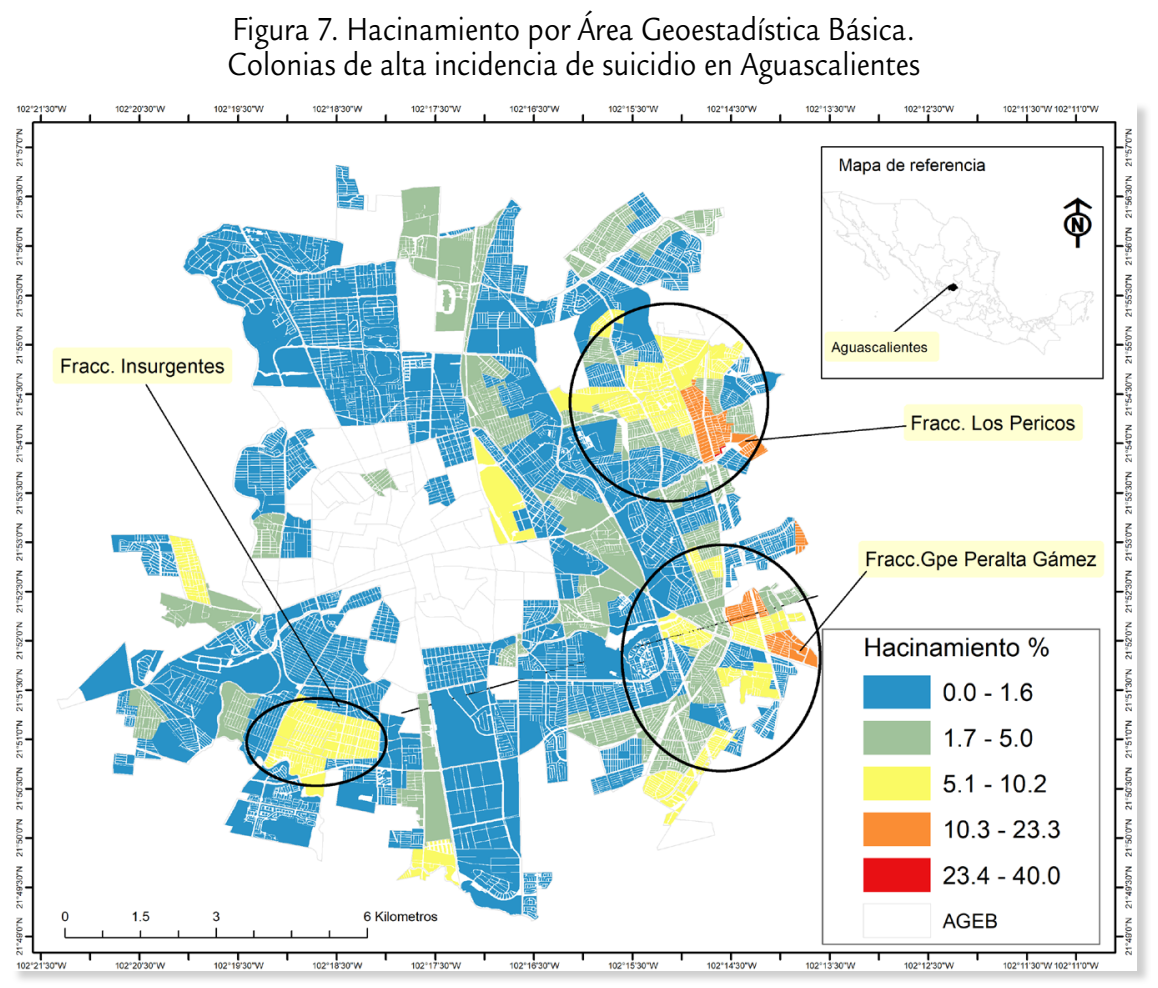

Fuente: CONEVAL (2010) y Hermosillo et al. (2015)

La representación de condiciones de hacinamiento revela un patrón que sugiere una correlación entre esta variable y la ubicación de los sitios de alta incidencia. La identificación de las áreas de alta incidencia se basa en la frecuencia de incidentes registrada por el Servicio Sicológico de atención en crisis y se documenta por el anuario IXTAB coordinado por Hermosillo et al. (2015). Este sistema captura entre otros datos la fecha del incidente, el día, la hora, el nombre completo de la persona con apellidos, sexo, edad, diagnóstico, domicilio, fraccionamiento y municipio. El proceso de georreferenciación de cada uno de los casos de suicidio y tentativa de suicidio se realiza mediante la plataforma Google Earth (Hermosillo et al., 2015, p. 22). Adicionalmente, la estimación revela que el $74,4 \%$ de las colonias en la zona considerada de alta incidencia de suicidio según los registros del Anuario del comportamiento suicida en Aguascalientes IXTAB (áreas marcadas con un círculo) están construidas bajo la modalidad de fraccionamiento (gated communities). Este modelo habitacional refleja un rasgo peculiar del modelo de desarrollo de la ciudad en las últimas cuatro décadas en el que el mercado inmobiliario ha capitalizado la llegada de flujos migratorios regionales ante el acelerado avance industrial de la ciudad, hecho que paradójicamente contrasta con el crecimiento observado en problemas de salud pública en esta ciudad como la evidencia empírica permite observar respecto al crecimiento de las tasas de mortalidad por suicidio en la entidad. Importante notar que se trata de una correlación no concluyente en términos de causalidad. 


\section{USO DE INTERNET Y TASAS DE MORTALIDAD POR SUICIDIO}

A continuación se analiza la correlación entre las tasas de suicidio y las tendencias de búsqueda en internet del término 'suicidio', un enfoque metodológico que busca aprovechar la creciente disponibilidad de información generada por los mecanismos masivos de comunicación. Con este propósito se utiliza la herramienta Google trends para indagar la importancia relativa del término 'suicidio' en búsquedas en esta plataforma durante un periodo de cinco años, lo que ofrece una perspectiva de las tendencias de largo plazo entre los 32 estados del país.

Posteriormente se utiliza este score para estimar la relación con la tasa de mortalidad por suicidio observada en cada entidad a partir de una ecuación bivariada de regresión lineal. La pregunta que este enfoque busca responder es: si existe una correlación estadísticamente significativa entre el comportamiento de búsqueda por términos que reflejan interés en el suicidio y el patrón de mortalidad expresado por las tasas de mortalidad observadas, un enfoque que ha sido estudiado con éxito en diversos países como Estados Unidos, Japón, Australia, Taiwán (Biddle et al., 2012; Sueki, Yonemoto, Takeshima e Inagaki, 2014). La Figura 8 muestra la estimación.

Figura 8. Relación tasa de mortalidad observada por estado 2013-2017 y Google trends score para el término 'suicidio' según entidad federativa en el periodo 2013-2017

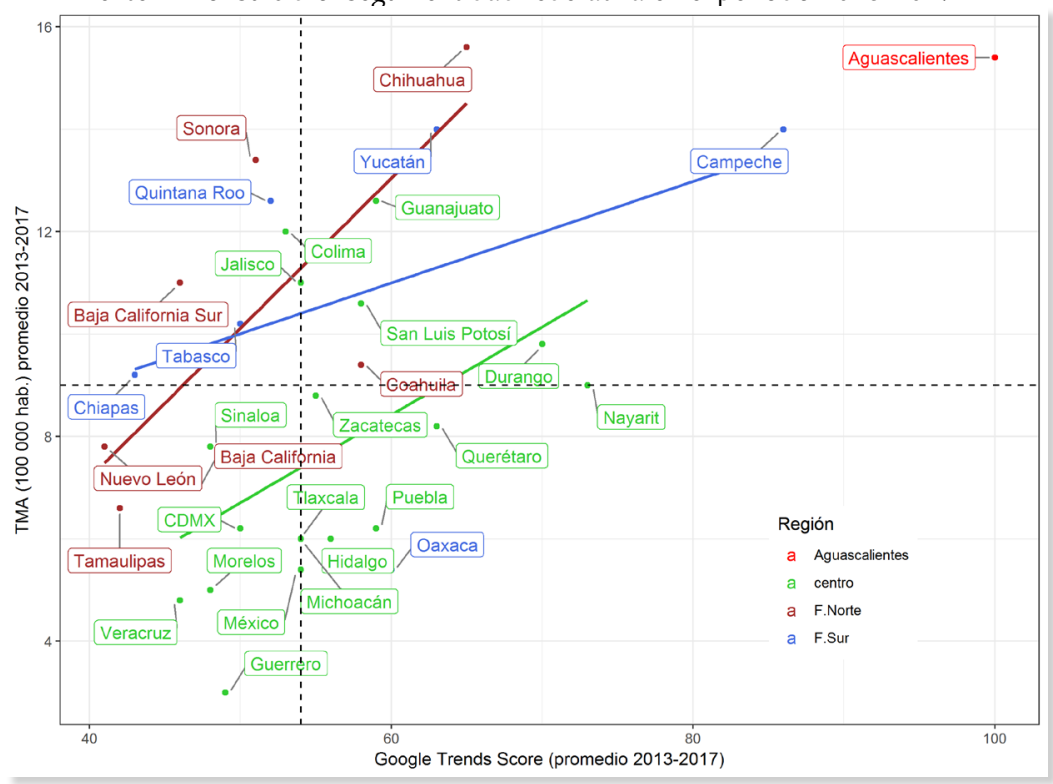

Nota: Líneas punteadas de referencia indican el valor promedio de cada variable en el periodo. Fuente: elaboración personal con datos de Google trends score 2013-2017. Tasas de mortalidad estimadas con datos de SINAIS (2013-2017)

Se encuentra una relación positiva y estadísticamente significativa ${ }^{10}$ que muestra que las entidades donde el término es más importante en las tendencias de búsqueda también exhiben las tasas de mortalidad por suicidio mayores. No obstante, podemos dis-

10 La especificación del modelo utilizado es la siguiente: $\mathrm{T}=\alpha+\beta$ Score $+\varepsilon$. Donde $\mathrm{T}$ es la tasa de mortalidad por suicidio estimada, Score es el indicador de la importancia relativa del término de búsqueda 'suicidio' para cada entidad federativa. $\mathrm{R}^{2}=0.2774, \mathrm{t}=3.394, \hat{y}=0.17368 \operatorname{Pr}(>|\mathrm{t}|)$ 0.00196. Por su parte la prueba Breusch-Pagan permite descartar posibles problemas de Heterocedasticidad ( $\mathrm{BP}=1.264, \mathrm{df}=1, \mathrm{p}$-value $=0.2609)$ donde H0: Homocedasticidad. Mientras la prueba Durbin-Watson para detectar autocorrelación indica que no se tiene este problema. DW $=1.6973$, $p$-value $=0.2016$. Finalmente, la prueba de normalidad de Shapiro-Wilk: $W=0.96558$, $p$-value $=0.3872$, indica que se tiene normalidad en los residuales. 
tinguir cuatro cuadrantes que muestran rasgos particulares de esta relación. El cuadrante I en términos cartesianos (superior derecho), es el que presenta una mayor intensidad en esta relación y se integra por aquellas entidades que muestran las mayores tasas de mortalidad en promedio, en conjunto con los niveles más elevados en el Google trends score en los cinco años considerados en la estimación. La composición de este cuadrante en términos de la distribución geográfica de las entidades que lo integran sugiere que este fenómeno tiene una distribución que alcanza las regiones del país de manera homogénea, siendo el caso extremo con ambos indicadores el estado de Aguascalientes.

La estimación permite distinguir también un subconjunto de entidades en donde se observan simultáneamente las menores tasas de mortalidad y los scores más pequeños; este es el caso de aquellos estados ubicados en el cuadrante 3 (inferior izquierdo).

La Figura 9 presenta las tendencias de búsqueda en Google para el caso particular del estado de Aguascalientes con dos series temporales, la primera de los últimos doce meses para mostrar el comportamiento más reciente y la segunda serie de cinco años en el periodo 2014-2019 con el propósito de identificar patrones cíclicos en la popularidad del término 'suicidio' en conjunto con un término asociado (tristeza). La selección de este término se propone con base en la literatura sobre determinantes desde la perspectiva de salud mental y parte del reconocimiento de los planteamientos de Durkheim sobre el nexo entre las emociones en tanto puente hacia las decisiones que los individuos toman.

Figura 9. Google trends score término tristeza, suicidio 2019

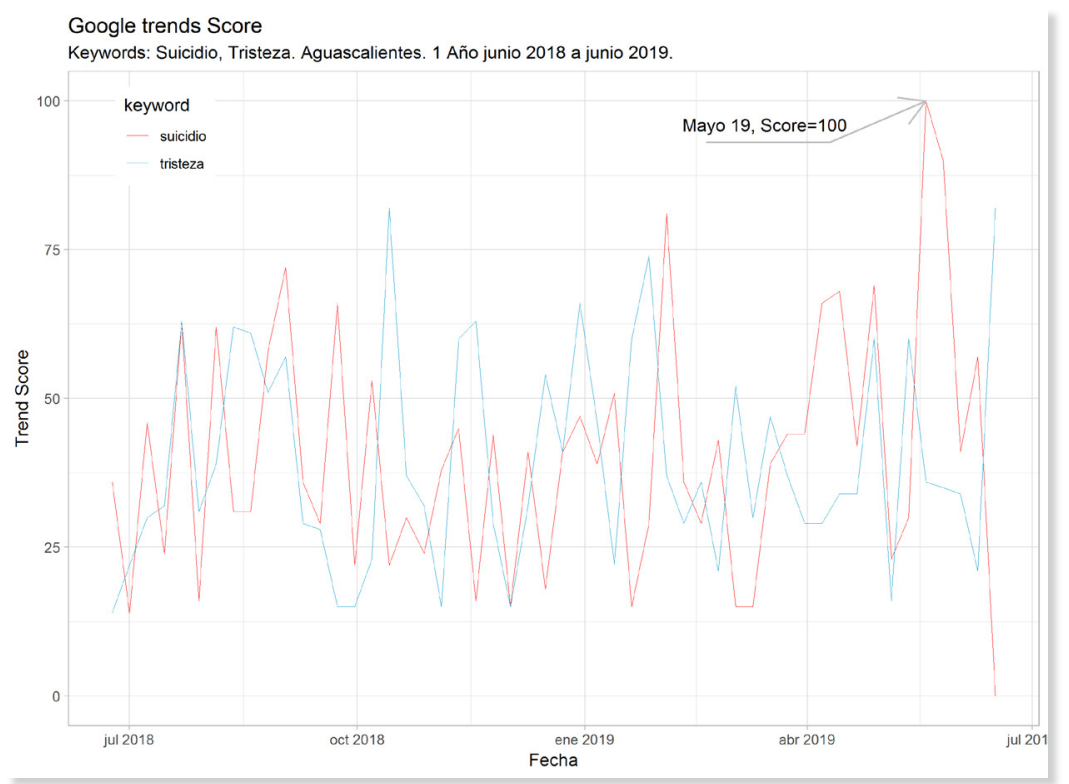

Fuente: elaboración personal con base en datos de Google trends score junio 2018-junio 2019

La estimación revela que es durante el mes de mayo en donde se ha tenido la mayor frecuencia de búsqueda del término suicidio durante los últimos doce meses. En segundo lugar, a pesar de que se observa cierto patrón de sincronía respecto al término tristeza con crestas que preceden al suicidio, para extraer la señal en la siguiente estimación se usa un filtro sobre la serie de tiempo para detectar la señal entre el ruido que generan las oscilaciones. El propósito del filtro es descubrir el patrón implícito predominante en la serie, lo que se logra al convertir la serie en una de menor frecuencia. La Figura 10 muestra este comportamiento. El impacto de este ajuste en el resultado, es que es posible de- 
tectar con mayor claridad la tendencia de largo plazo, por ejemplo, las crestas o momentos de mayor popularidad y meses de menor popularidad de los términos estudiados.

La serie de largo plazo permite corroborar un patrón de mayor frecuencia para el término suicidio en los meses de primavera abril, mayo y junio con cinco de los seis mayores registros en el score. Al controlar por el ruido de las oscilaciones se encuentra que de hecho las series muestran patrones que convergen alrededor de los meses de invierno (diciembre), un rasgo que merece un análisis con mayor detalle por lo que se propone como elemento de estudio en la agenda de investigación sobre el tema.

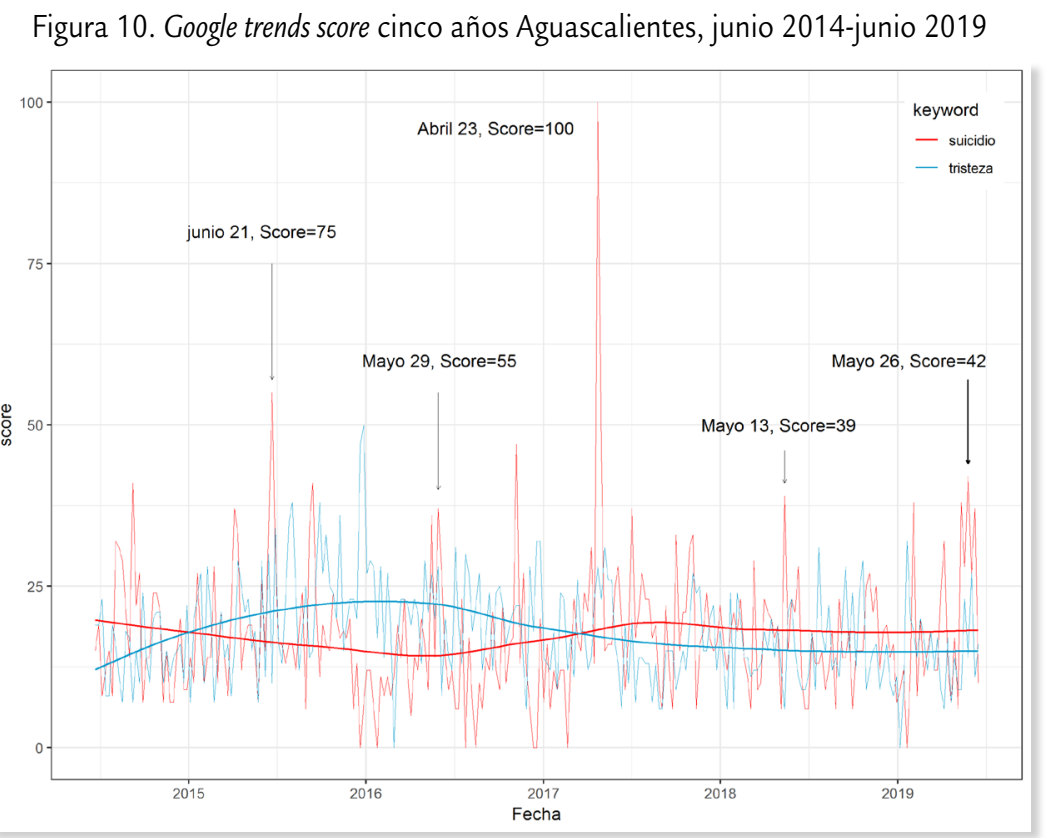

Fuente: elaboración personal con base en datos de Google trends score

\section{DISCUSIÓN}

Los resultados de la presente investigación aportan información actualizada con desagregación espacial útil para el diseño de estrategias de monitoreo y permiten dar seguimiento a iniciativas de investigación pioneras para México en línea con el interés planteado por la Organización Mundial de la salud (WHO, 2014) a través del plan de acción sobre salud mental.

El escenario base sobre tasas de mortalidad indica que los estados de Chihuahua y Aguascalientes son los de mayor mortalidad actualmente; estos resultados contrastan con las estimaciones de (Dávila-Cervantes, Torres y Casique, 2015), quienes ubican el foco de atención en entidades del sur del país. Por su parte la incorporación de series temporales aporta una perspectiva única sobre el crecimiento del suicidio en México en las últimas dos décadas y permite explicar el reposicionamiento observado a escala regional hacia entidades de alto crecimiento económico como Aguascalientes.

Si bien la estimación revela un claro patrón de mayor incidencia para el sexo masculino, sin excepción en los 32 estados del país y este es un resultado consistente con la evidencia reportada por estudios previos a cargo de Borges et al. (2010), la magnitud de la brecha por sexo es una aportación del análisis que se sugiere retomar para el diseño de estrategias preventivas focalizadas. 
La dimensión temporal muestra que el estado de Aguascalientes tiene el mayor crecimiento de este fenómeno social en el periodo estudiado, lo que sugiere un problema persistente de largo plazo en la entidad. El planteamiento metodológico tiene especial atención en evitar el sesgo derivado de la dinámica demográfica en cada entidad, particularmente inducido por el componente migratorio, un factor con influencia mayor en entidades como Quintana Roo. En este sentido, el análisis de la brecha respecto a la trayectoria de crecimiento del resto de causas de muerte permite aislar el comportamiento del suicido.

El análisis a escala de Zona Metropolitana ofrece una perspectiva de mayor precisión y posibilita identificar las áreas urbanas de alta concentración en México. Así, se encuentra que la ZM de Aguascalientes ocupa la primera posición entre las 59 ZM que integran el país, rasgo que confirma la severidad de este fenómeno social en esta zona. A pesar de su distribución heterogénea en el territorio nacional, un rasgo común entre las ZM de alta concentración es que se trata de espacios urbanos que han experimentado flujos migratorios intensivos y un elevado ritmo de crecimiento económico en años recientes. Una vez que se aborda el caso particular de Aguascalientes los resultados permitieron identificar a la población entre 12 y 32 años de edad como un grupo de alto riesgo con las implicaciones propias sobre el tejido social en diversas áreas. Este resultado consistente con la observación de la organización mundial de la salud WHO (2017b) y diversos estudios empíricos como Zalsman (2019), sobre la magnitud del fenómeno en edades tempranas, lo que sugiere otro insumo para el diseño de intervenciones preventivas. Se detectó que la causa específica de mayor importancia es el ahorcamiento (X70), seguida de envenenamiento (X60-64) y disparo de arma de fuego (X72-X75); lo anterior al margen de los sesgos generados por las deficiencias en el registro de causas por factores externos.

Los resultados en esta dimensión, contrastan con las tendencias observadas en países de la región de Norteamérica particularmente con el caso de Estados Unidos donde el método de mayor frecuencia es disparo de arma de fuego (Ajdacic-Gross et al., 2008). De manera complementaria, investigaciones que se enfocan en la predilección por los métodos para consumar el suicidio en otras regiones del mundo como Kõlves, McDonough, Crompton y de Leo (2018), advierten de un comportamiento diferencial por sexo. Este nivel de detalle es un área de oportunidad identificada por este trabajo que requiere validación empírica formal y se propone para avanzar en la agenda de investigación de este tema ya que puede ayudar en los esfuerzos de prevención al informar sobre diferencias de comportamiento por sexo según causa específica.

Por su parte, los hallazgos del análisis sobre la distribución de los casos por mes contrastan con la evidencia de estudios previos que reportan una mayor incidencia en los meses de invierno. Acorde con Thorson y Kasworm (2007), esta hipótesis asume un mecanismo en el que la reducción en la frecuencia de interacción social durante los meses de invierno puede tener un efecto negativo sobre cómo un individuo deprimido enfrenta el stress, lo que a su vez incrementa el riesgo de suicidio.

En contraposición a esa hipótesis, los resultados de la presente investigación que capturan el comportamiento por mes en un total de 20 años, muestran que mientras condiciones depresivas pueden presentarse en los meses de invierno, como da cuenta la convergencia entre los términos suicidio y tristeza, la acción o concreción de las ideas suicidas de hecho ocurre en los meses de mayo y junio. Al respecto el manuscrito documenta que el score máximo para el término de búsqueda 'suicidio' en Aguascalientes ocurre regularmente en los meses de mayo y junio, meses que también registran la mayor 
incidencia de suicidio para el estado, resultado que es consistente con los patrones detectados por estudios recientes en el contexto de climas tropicales (Akkaya-Kalayci et al., 2017; Gao et al., 2019).

Una posible explicación documentada sobre este comportamiento por estudios previos, es que la elevación de la temperatura puede incrementar el comportamiento impulsivo y agresivo de las personas a través de elevados niveles de serotonina (receptores 5-HT), un neurotransmisor que se vincula entre otros procesos con las variaciones en el estado de ánimo (Zhang y Tao, 2011; Żmudzka, Sałaciak, Sapa y Pytka, 2018).

La perspectiva con escala intraurbana que se aborda en la tercera fase de la investigación, indica la importancia del diseño de políticas públicas que fomenten la creación de espacios urbanos saludables. Los resultados dan cuenta de condiciones sociales asociadas a un proceso de expansión inmobiliaria orientado a la creación de unidades habitacionales periféricas que albergan a la población con los mayores niveles de hacinamiento, un elemento de contexto considerado entre los factores que inciden en el desarrollo de afecciones de salud mental, propias de la fenomenología del suicidio. La evidencia del análisis sobre esta vertiente, concuerda con la propuesta clásica de Durkheim (1951), en donde las fuerzas sociales constituyen factores causales, en especial rasgos propios de un estado social de anomia, en donde al parecer la velocidad de los cambios en el entorno económico ocurridos durante las últimas dos décadas en estados como Aguascalientes se han acompañado por consecuencias en el tejido social, lo que es consistente con los problemas detectados por autores como Enríquez-Aranda (1995) y particularmente con las apreciaciones de Hermosillo et al. (2015).

No obstante, se reconoce que el estudio de esta dimensión con mayor nivel de desagregación, rebasa los límites de la presente investigación y es preciso anotar que la evidencia empírica por estudios realizados en países de la región Latinoamérica, como los de Leveau, et al., (2019), quienes abordan el caso de Argentina, sugiere que la dirección y la magnitud de la relación entre hacinamiento y suicidio es sensible a la definición del indicador de hacinamiento estudiado, así como de las variables de control empleadas para modelar el fenómeno, por lo que se propone que la validación empírica de este patrón requiere de estudios de campo adicionales para obtener información de respaldo, en particular contar con información georreferenciada de los casos ocurridos en un periodo de tiempo determinado es deseable.

El estudio buscó adicionalmente la aplicación de herramientas innovadoras de recopilación de datos dirigidas al posicionamiento que el uso de medios masivos de comunicación entre la población tiene actualmente, como es el uso de internet, específicamente a través de plataformas de búsqueda como Google. Los resultados de esta aplicación concuerdan con estimaciones recientes en el contexto de países como Estados Unidos (Ma-Kellams et al., 2016), en los que se demuestra que la correlación entre los patrones de búsqueda registrados por herramientas como Google trends y las tasas de mortalidad observadas por suicidio, de hecho, revelan una función dual del uso de internet, en tanto que actúa como un medio facilitador, así como una herramienta preventiva potencial.

\section{CONCLUSIONES}

La presente investigación documentó la trayectoria temporal y distribución del suicidio en México con énfasis en el estado de Aguascalientes durante el periodo 1998-2017 a partir de la incorporación de elementos del contexto territorial, una propuesta que 
explícitamente complementa el abordaje tradicional del suicidio como un mero síntoma de un desequilibrio siquiátrico.

Las acciones de política pública emprendidas en México para atender el suicidio se han concentrado hasta el momento en una aproximación tradicional del fenómeno con acciones integradas de prevención y tratamiento de adicciones y aquellas bajo la perspectiva de salud mental mediante el reforzamiento del sistema de vigilancia epidemiológica. No obstante, en la presente investigación se aporta evidencia que demuestra que ese enfoque, si bien necesario, no es suficiente para comprender el crecimiento observado en este fenómeno social. Por este motivo la realización de investigaciones con un enfoque territorial desde aproximaciones multidisciplinarias se propone como una tarea permanente. Esto implica entre otros aspectos, avanzar en colaboración para la difusión de resultados de investigación en los diferentes niveles de la sociedad, no solo en el ámbito académico.

Desde la perspectiva de la política pública, la carencia de estrategias efectivas que incidan en el control del avance de este fenómeno en el caso de Aguascalientes se manifiesta por el hecho de que las tasas de suicidio en el periodo de 20 años estudiado, no solo no han experimentado una disminución, sino que su crecimiento ha seguido una trayectoria de continuo ascenso que ha superado el comportamiento en cualquier otra entidad del país.

El trabajo permitió mostrar también el potencial que el uso de grandes volúmenes de datos en el contexto de herramientas de comunicación actuales, como Google, ofrecen para estudiar temas ligados a determinantes sociales, donde las necesidades de pertenencia del individuo y su expresión por diversos medios de comunicación pueden constituir un elemento clave para comprender este fenómeno.

\section{REFERENCIAS}

Abrutyn, S. y Mueller, A.S. (2014). The Socioemotional Foundations of Suicide: A Microsociological View of Durkheim's Suicide. Sociological Theory, 32(4), 327-351. DOI https:// doi.org/10.1177/0735275114558633

Aguilar, A.G. y López, F.M. (2016). Espacios de pobreza en la periferia urbana y suburbios interiores de la Ciudad de México: Las desventajas acumuladas. EURE, 42, 5-29.

Ajdacic-Gross, V.; Weiss, M.; Ring, M.; Hepp, U.; Bopp, M.; Gutzwiller, F. y Rössler, W. (2008). Methods of suicide: International suicide patterns derived from the WHO mortality database. Bulletin of the World Health Organisation, 86, 726-732. DOI https:// doi.org/10.1590/S0042-96862008000900017

Akkaya-Kalayci, T.; Vyssoki, B.; Winkler, D.; Willeit, M.; Kapusta, N.; Dorffner, G. y Özlü-Erkilic, Z. (2017). The effect of seasonal changes and climatic factors on suicide attempts of young people. BMC Psychiatry, 17, 365. DOI https://doi.org/10.1186/ s12888-017-1532-7

Araya, R.; Lewis, G.; Rojas, G. y Fritsch, R. (2003). Education and income: Which is more important for mental health? Journal of Epidemiology and Community Health, 57(7), 501-505. DOI https://doi.org/10.1136/jech.57.7.501

Bando, D.H.; Moreira, R.S.; Pereira, J.C.R. y Barrozo, L.V. (2012). Spatial clusters of suicide in the municipality of São Paulo 1996-2005: An ecological study. BMC Psychiatry, 12, 124. DOI https://doi.org/10.1186/1471-244X-12-124

Biddle, L.; Gunnell, D., Owen-Smith, A., Potokar, J., Longson, D., Hawton, K., Kapur, N. y Donovan, J. (2012). Information sources used by the suicidal to inform choice of 
method. Journal of Affective Disorders, 136(3), 702-709. DOI https://doi.org/10.1016/j. jad.2011.10.004

Bonnefoy, X. (2007). Inadequate housing and health: An overview. International Journal of Environment and Pollution, 30(3-4). DOI https://doi.org/10.1504/IJEP.2007.014819

Borges, G.; Orozco, R.; Benjet, C. y Medina-Mora, M. E. (2010). Suicidio y conductas suicidas en México: Retrospectiva y situación actual. Salud Pública de México, 52, 292-304.

Bruns, R.; Dunkel, J. y Offel, N. (2019). Learning of Complex Event Processing Ruleswith Genetic Programming. Expert Systems with Applications, 129, 186-199.

Bunge, M. (2012). Wealth and well-being, economic growth, and integral development. International Journal of Health Services, 42(1), 65-76.

Chandler, V. (2018). Google and suicides: What can we learn about the use of internet to prevent suicides? Public Health, 154, 144-150. DOI https://doi.org/10.1016/j.puhe.2017.10.016

Cole, H.; Shokry, G.; Connolly, J.J.T.; Pérez-Del-Pulgar, C.; Alonso, J. y Anguelovski, I. (2017). Can Healthy Cities be made really healthy? The Lancet. Public Health, 2(9), e394-e395. DOI https://doi.org/10.1016/S2468-2667(17)30166-4

Consejo Nacional de Evaluación de la Política de Desarrollo Social, CONEVAL (2010). Base de datos rezago social de todas las AGEB zonas urbanas. Recuperado de https://www. coneval.org.mx/Medicion/IRS/Paginas/Rezago_social_AGEB_2010.aspx

Consejo Nacional de Población, CONAPO. (2010). Delimitación de Zonas metropolitanas. SEGOB. Recuperado de http://www.conapo.gob.mx/es/CONAPO/Zonas_metropolitanas_2010

Cutler, D.; Deaton, A. y Lleras-Muney, A. (2006). The Determinants of Mortality. Journal of Economic Perspectives, 20(3), 97-120. DOI https://doi.org/10.1257/jep.20.3.97

Dávila-Cervantes, C.; Torres, M. y Casique, I. (2015). Análisis del impacto de la mortalidad por suicidios en México, 2000-2012. Salud Colectiva, 11, 471. DOI https://doi. org/10.18294/sc.2015.784

Diario Oficial de la Federació (2012a). Norma Oficial Mexicana NOM-024-SSA3-2012, Sistemas de información de registro electrónico para la salud. Intercambio de información en salud. Secretaria de Gobernación. Recuperado de https://dof.gob.mx/nota_detalle.php?codigo $=5280847 \&$ fecha $=30 / 11 / 2012$

Diario Oficial de la Federación (2012b). Norma Oficial Mexicana NOM-035-SSA3-2012. En materia de información en salud. Secretaria de Gobernación. Recuperado de http://dof. gob.mx/nota_detalle.php?codigo=5280848\&fecha=30/11/2012

Djira, G.; Schaarschmidt, F. y Fayissa, B. (2010). Inferences for Selected Location Quotients with Applications to Health Outcomes. Geographical Analysis, 42, 288-300. DOI https://doi.org/10.1111/j.1538-4632.2010.00794.x

Douthwaite, R. (2011). The Growth Illusion: How economic growth has enriched the few, impoverished the many and endangered the planet. The lilliput press.

Durkheim, É. (1951). Suicide: A Study in Sociology. Free Press. Recuperado de https://books. google.com.mx/books?id=ZoraAAAAMAAJ

Eibenschutz, R. y Goya, C. (2009). Estudio de la integración urbana y social en la expansión reciente de las ciudades en México, 1996-2006: Dimensión, características y soluciones. Secretaría de Desarrollo Social. Recuperado de https://books.google.com.mx/books?id=c3IOQgAACAAJ Enriquez-Aranda, R. (1995). Desarrollo Urbano en la ciudad de Aguascalientes. Investigación y ciencia, 30-35.

Evans, G.; Wells, N. y Moch, A. (2003). Housing and Mental Health: A Review of the 
Evidence and a Methodological and Conceptual Critique. Journal of Social Issues, 59, 475-500. https://doi.org/10.1111/1540-4560.00074

Franco Muñoz, R.; López Flores, C.; Ruiz León, C.; Santiago García, L.E.; Mendoza Palacios, S.; García, J.; Mendoza Romo, A.F. y Peralta Marques, L. (2004). Funcionalidad de la vivienda en los fraccionamientos habitacionales urbanos de tipo popular en la ciudad de Aguascalientes. Investigación y ciencia, 12(30), 29-38.

Gao, J.; Cheng, Q.; Duan, J.; Xu, Z.; Bai, L.; Zhang, Y.; Zhang, H.; Wang, S.; Zhang, Z. y $\mathrm{Su}, \mathrm{H}$. (2019). Ambient temperature, sunlight duration, and suicide: A systematic review and meta-analysis. The Science of the total environment, 646, 1021-1029. DOI https:// doi.org/10.1016/j.scitotenv.2018.07.098

García-Rábago, H.; Sahagún-Flores, J.; Ruiz-Gómez, A.; Sánchez-Ureña, G.; Tirado-Vargas, J. y González-Gámez, J. (2010). Factores de riesgo, asociados a intento de suicidio, comparando factores de alta y baja letalidad. Revista de Salud Pública, 12, 713-721. DOI https://doi.org/10.1590/S0124-00642010000500002

Google (2018). Google trends. Recuperado dehttps://trends.google.com.mx/trends/?geo=MX Hermosillo, A.E.; Vacio, M.Á.; Ponce de León, A.; Ortega, S. y Macías, G. (2015). Anuario del comportamiento suicida en el estado de Aguascalientes. UAA. Recuperado de https://editorial.uaa.mx/docs/anuario_suicida_ags.pdf

Hernández-Bringas, H.H. y Flores-Arenales, R. (2011). El suicidio en México. Papeles de población, 17, 69-101.

Higgins, T.S.; Wu, A.W.; Sharma, D.; Illing, E.A.; Rubel, K. y Ting, J.Y. (2020). Correlations of Online Search Engine Trends With Coronavirus Disease (COVID-19) Incidence: Infodemiology Study. JMIR Public Health and Surveillance, 6(2), e19702. DOI https://doi. org/10.2196/19702

Howard, P.; Woolley, S. y Ryan, C. (2018). Algorithms, bots, and political commu-nication in the US 2016 election: The challenge of automated political communication forelection law and administration. Journal of Information Technology \& Politics, 15(2).

lemmi, V.; Bantjes, J.; Coast, E.; Channer, K.; Leone, T.; McDaid, D.; Palfreyman, A.; Stephens, B. y Lund, C. (2016). Suicide and poverty in low-income and middle-income countries: A systematic review. The Lancet. Psychiatry, 3(8), 774-783. DOI https://doi. org/10.1016/S2215-0366(16)30066-9

Joiner, T. (2007). Why People Die by Suicide. Bibliovault OAI Repository, the University of Chicago Press.

Jusidman, C. (2012). Contextos urbanos, pobreza y violencia. En La pobreza urbana en México: Nuevos enfoques y retos emergentes para la acción pública. El Colegio de la Frontera Norte/Juan Pablos.

Keller, T. y Ulrike, K. (2019). Social Bots in Election Campaigns: Theoretical, Empir-ical, and Methodological Implications. Political Communication, 36(1), 171-189.

Kõlves, K.; McDonough, M.; Crompton, D. y de Leo, D. (2018). Choice of a suicide method: Trends and characteristics. Psychiatry Research, 260, 67-74. DOI https://doi. org/10.1016/j.psychres.2017.11.035

Leveau, C.M., Guevel, C. y Alazraqui, M. (2019). Diferenciales intra-urbanos del suicidio: El rol de la fragmentación social en Argentina. Cien Saude Colet. Recuperado de http://www.cienciaesaudecoletiva.com.br/artigos/diferenciales-intraurbanos-del-suicidio-el-rol-de-la-fragmentacion-social-en-argentina/17389 
Libertun de Duren, N.R. (2018). The social housing burden: Comparing households at the periphery and the centre of cities in Brazil, Colombia, and Mexico. International Journal of Housing Policy, 18(2), 177-203. DOI https://doi.org/10.1080/19491247.2017.1298366

Ma-Kellams, C.; Or, F.; Baek,J.H.yKawachi, I. (2016). Rethinking Suicide Surveillance: Google Search Data and Self-Reported Suicidality Differentially Estimate Completed Suicide Risk. Clinical Psychological Science, 4. DOI https://doi.org/10.1177/2167702615593475

Manzanares Rivera, J.L. (2016). Calidad de los recursos hídricos en el ontexto de la actividad económica y patrones de salud en Sonora, México. Salud Colectiva, 3(12), 397-414.

Manzanares Rivera, J.L. (2017). Distribución espacial de egresos hospitalarios de casos por infección vírica por picadura de mosquito en México entre 2004 y 2014. Rev Panam Salud Publica, 41(30), 8.

Marí-Dell'Olmo, M.; Novoa, A.M.; Camprubí, L.; Peralta, A.; Vásquez-Vera, H.; Bosch, J.; Amat, J.; Díaz, F.; Palència, L.; Mehdipanah, R.; Rodríguez-Sanz, M.; Malmusi, D. y Borrell, C. (2017). Housing Policies and Health Inequalities. International Journal of Health Services, 47(2), 207-232. DOI https://doi.org/10.1177/0020731416684292

Martínez Salgado, C. (2010). Población y salud mental en México. Reflexiones y un ejercicio de aproximación mediante las variaciones de la mortalidad por suicidio. Estudios demográficos y urbanos, 25, 663-712.

Meyer, C.; Irani, T.; Hermes, K. y Yung, B. (2017). The Complexity of Suicide Motivation (pp. 121-145). En Explaining Suicide. Patterns, Motivations, and What Notes Reveal. DOI https://doi.org/10.1016/B978-0-12-809289-7.00006-3

Mok, K.; Jorm, A.F. y Pirkis, J. (2015). Suicide-related Internet use: A review. The Australian and New Zealand Journal of Psychiatry, 49(8), 697-705. DOI https://doi. org/10.1177/0004867415569797

Montejano Escamilla, J.A.; Caudillo Cos, C.A. y Cervantes Salas, M. (2018). Vivienda de interés social, segregación residencial y accesibilidad: Análisis de 121 conjuntos urbanos en el arco nororiente del Valle de México, 2001-2010. Estudios demográficos y urbanos, 33, 187-224.

Organización Panamericana de la Salud, OPS. (2016). Prevención de la conducta suicida. WHO. Recuperado de https://iris.paho.org/bitstream/handle/10665.2/31167/978927 5319192-spa.pdf? sequence $=1$ \&isAllowed $=y$

Ortiz, R.M. (2017). Apuntes sobre la participación del sector privado en el desarrollo de vivienda social en México. Archivio di Studi Urbani e Regionali, XLVIII(118 suppl.), 57-77. DOI https://doi.org/10.3280/ASUR2017-118S04

Pepin, C.; Muckle, G.; Moisan, C.; Forget-Dubois, N. y Riva, M. (2018). Household overcrowding and psychological distress among Nunavik Inuit adolescents: A longitudinal study. International Journal of Circumpolar Health, 77, 1541395. DOI https://doi.org/10.10 80/22423982.2018.1541395

Pompili, M. (2018). Reflections of a Committed Suicidologist (pp. 13-30). En Phenomenology of Suicide: Unlocking the Suicidal Mind. DOI https://doi.org/10.1007/978-3-319-47976-7_2

Rasse, A. (2015). Juntos pero no revueltos: Procesos de integración social en fronteras residenciales entre hogares de distinto nivel socioeconómico. EURE, 41, 125-143.

Rizvi, S.J.; Iskric, A.; Calati, R. y Courtet, P. (2017). Psychological and physical pain as predictors of suicide risk: Evidence from clinical and neuroimaging findings. Current Opinion in Psychiatry, 30(2), 159-167. DOI https://doi.org/10.1097/YCO.0000000000000314

Rojas, M.C.; Meichtry, N.C.; Ciuffolini, M.B.; Vázquez, J.C. y Castillo, J. (2008). Repen- 
sando de manera holística el riesgo de la vivienda urbana precaria para la salud: Un análisis desde el enfoque de la vulnerabilidad sociodemográfica. Salud Colectiva, 4(2). DOI https://doi.org/10.18294/sc.2008.341

Rosales, C.B.; Carvajal, S. y de Zapien, J.E.G. (2016). Emergent Public Health Issues in the US-Mexico Border Region. Frontiers in Public Health, 4, 93-93. DOI https://doi. org/10.3389/fpubh.2016.00093

Rufino, M.B. (2015). O imobiliário como frente de expansão da metrópole: Contradices na produção do espado do Porto das Dunas. EURE, 41, 69-90.

Sánchez-Cervantes, F.; Serrano-González, R. y Márquez-Caraveo, M.E. (2015). Suicidios en menores de 20 años. México 1998-2011. Salud Mental, 38(5), 379-389.

Schlimme, J.E. (2018). A Phenomenological Approach to Suicidal Mental Life (pp. 3137). En M. Pompili (Ed.) Phenomenology of Suicide: Unlocking the Suicidal Mind. Springer International Publishing. DOI https://doi.org/10.1007/978-3-319-47976-7_3

Shneidman, E.S. (1993). Suicide as psychache. The Journal of Nervous and Mental Disease, 181(3), 145-147. DOI https://doi.org/10.1097/00005053-199303000-00001

Sistema nacional de información en Salud, SINAIS. (2015). Base de datos sobre defunciones. Secretaría de Salud. Recuperado de http://www.dgis.salud.gob.mx/contenidos/sinais/s_seed.html

Sistema Nacional de Información de Salud SINAIS (2017). Base de datos defunciones. Secretaría de Salud. Recuperado de http://www.dgis.salud.gob.mx/contenidos/sinais/s_seed.html

Sistema Nacional de Información de Salud SINAIS (1998). Base de datos defunciones. Recuperado de http://www.dgis.salud.gob.mx/contenidos/sinais/s_seed.html

Soreni, N.; Cameron, D.H., Streiner, D L.; Rowa, K. y McCabe, R.E. (2019). Seasonality Patterns of Internet Searches on Mental Health: Exploratory Infodemiology Study. JMIR Mental Health, 6(4), e12974. DOI https://doi.org/10.2196/12974

Sueki, H.; Yonemoto, N.; Takeshima, T. e Inagaki, M. (2014). The Impact of Suicidality-Related Internet Use: A Prospective Large Cohort Study with Young and Middle-Aged Internet Users. PloS one, 9, e94841. DOI https://doi.org/10.1371/journal.pone.0094841

Thorson, J. A. y Kasworm, C. (2007). Sunshine and suicide: Possible influences of climate on behavior. Death Education, 8(sup001), 125-136. DOI https://doi. org/10.1080/07481188408252493

Urria-Yánez, I. (2018). Trayectorias de hacinamiento y salud mental: El impacto asimétrico del hacinamiento físico sobre síntomas depresivos en Chile. Santiago: Editorial Express.

Van Orden, K.A.; Witte, T.K.; Cukrowicz, K.C.; Braithwaite, S.R.; Selby, E.A. y Joiner, T.E.J. (2010). The interpersonal theory of suicide. Psychological Review, 117(2), 575-600. DOI https://doi.org/10.1037/a0018697

World Health Organization (2014). Preventing suicide: A global imperative. World Health Organization.

World Health Organization. (2017a). Ten years in public health, 2007-2017: Report by Dr Margaret Chan, Director-General, World Health Organization. World Health Organization; WHO IRIS. Recuperado de https://apps.who.int/iris/handle/10665/255355

World Health Organization WHO (2017b). WHO Mortality Database (p. 2). World Health Organization. Recuperado de https://www.who.int/mental_health/suicide-prevention/country-profiles/MEX.pdf?ua=1

World Health Organization. (2018). Preventing suicide: A community engagement toolkit. 
World Health Organization. WHO IRIS. Recuperado de https://apps.who.int/iris/ handle/10665/272860

Zalsman, G. (2019). [Suicide: Epidemiology, Etiology, Treatment And Prevention]. Harefuah, 158(7), 468-472.

Zhang, G. y Tao, R. (2011). Enhanced responsivity of 5-HT(2A) receptors at warm ambient temperatures is responsible for the augmentation of the. Neuroscience Letters, 490(1), 68-71. DOI https://doi.org/10.1016/j.neulet.2010.12.028

Żmudzka, E.; Sałaciak, K.; Sapa, J. y Pytka, K. (2018). Serotonin receptors in depression and anxiety: Insights from animal studies. Life Sciences, 210, 106-124. DOI https://doi. org/10.1016/j.Ifs.2018.08.050

José Luis Manzanares Rivera es Profesor Investigador Adscrito al Departamento de Estudios Urbanos y Medio Ambiente en El Colegio de la Frontera Norte. Es miembro del Sistema Nacional de Investigadores. Sus intereses de investigación incluyen la aplicación de Sistemas de Información Geográfica para el estudio de los patrones territoriales de desarrollo. Sus áreas de especialización en docencia son: métodos cuantitativos y estadística aplicada. Departamento de Estudios Urbanos y Medio Ambiente. El Colegio de la Frontera Norte. Av. Reforma, Local 1, 2 y 3 (Centro Corporativo Norte) Col. del Rosario, (84020), Nogales, Son, México, jlmanzanares@colef.mx, ORCID https://orcid.org/0000-0003-3394-4967 